\title{
Al-guided use of balanced PPARa/Y dual agonist finetunes macrophage responses in inflammatory bowel disease
}

\section{Gajanan Katkar}

University of California, San Diego

Ibrahim Sayed

University of California, San Diego

Mahitha Shree Anandachar

University of California San Diego

Vanessa Castillo

University of California San Diego

Vidales Eleadah

University of California San Diego

Daniel Toobian

University of California San Diego

Fatima Usmani

University of California San Diego

Joseph Sawires

University of California San Diego

Geoffray Leriche

University of California San Diego

Jerry Yang

University of California, San Diego https://orcid.org/0000-0002-8423-7376

William Sandborn

University of California San Diego

Soumita Das

University of California San Diego

Debashis Sahoo

University of California, San Diego https://orcid.org/0000-0003-2329-8228

Pradipta Ghosh ( $\nabla$ prghosh@ucsd.edu )

University of California San Diego 
Keywords: Boolean Implication Network, Inflammatory Bowel Disease (IBD), Reactive Oxygen Species (ROS), Macrophage, Bacterial clearance, Citrobacter, PPARa/g-dual agonists, PAR5359

Posted Date: March 19th, 2021

DOI: https://doi.org/10.21203/rs.3.rs-243294/v1

License: (c) (1) This work is licensed under a Creative Commons Attribution 4.0 International License. Read Full License 


\section{AI-guided use of balanced PPARa/ $\gamma$ dual agonist finetunes macrophage responses in inflammatory bowel disease}

Authors: Gajanan D. Katkar ${ }^{1}$, Ibrahim M. Sayed ${ }^{2 \$}$, Mahitha Shree Anandachar ${ }^{2}$, Vanessa Castillo ${ }^{1}$, Vidales, Eleadah ${ }^{1}$, Daniel Toobian ${ }^{1}$, Fatima Usmani ${ }^{2}$, Joseph R. Sawires ${ }^{3}$, Geoffray Leriche ${ }^{3}$, Jerry Yang $^{3}$, William J. Sandborn ${ }^{4 *}$, Soumita Das ${ }^{2 *}$, Debashis Sahoo ${ }^{5,6,7 *}$ and Pradipta Ghosh ${ }^{1,4,7,8 *}$

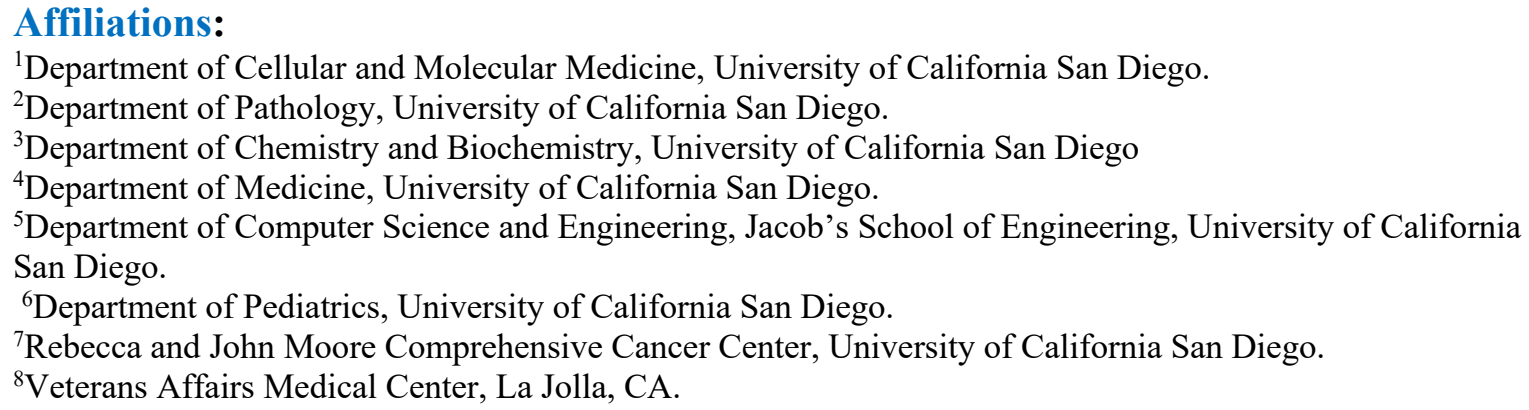

Alternative affiliation: ${ }^{\circledR}$ Department of Medical Microbiology and Immunology, Faculty of Medicine, Assiut University, Egypt.

Conflict of interest statement: S.D, D.S and P.G have a patent on the methodology. Barring this, the authors have declared that no conflict of interest exists.

\section{*Correspondence to:}

William J. Sandborn, M.D.; Professor, Department of Medicine, University of California San Diego; 9500 Gilman Drive, MC 0956, La Jolla, CA 92093-0831.

Phone: 858-657-5331, Email: wsandborn@health.ucsd.edu

Soumita Das, Ph.D.; Associate Professor, Department of Pathology, University of California, San Diego; 9500 Gilman Drive, George E. Palade Bldg, Rm 256, 239; La Jolla, CA 92093.

Phone: 858-246-2062 (office): Email: sodas@ucsd.edu

Debashis Sahoo, Ph.D; Assistant Professor, Department of Pediatrics, University of California San Diego; 9500 Gilman Drive, MC 0730, Leichtag Building 132; La Jolla, CA 92093-0831.

Phone: 858-246-1803: Fax: 858-246-0019: Email: dsahoo@ucsd.edu

Pradipta Ghosh, M.D.; Professor, Departments of Medicine, and Cell and Molecular Medicine, University of California San Diego; 9500 Gilman Drive (MC 0651), George E. Palade Bldg, Rm 232, 239; La Jolla, CA 92093. Phone: 858-822-7633: Fax: 858-822-7636: Email: prghosh@ucsd.edu 
54 A computational platform, the Boolean network explorer $(B o N E)$, has recently been developed to infuse AI-enhanced precision into drug discovery; it enables querying and navigating invariant Boolean Implication Networks of disease maps for prioritizing high-value targets. Here we used BoNE to query an Inflammatory Bowel Disease (IBD)-map and prioritize two nuclear receptors, PPAR $\alpha / \gamma$. Balanced agonism of PPAR $\alpha / \gamma$ was predicted to impact macrophage processes, ameliorate colitis in network-prioritized animal models, 'reset' the gene expression network from disease to health, and achieve a favorable therapeutic index that tracked other FDA-approved targets. Predictions were validated using a balanced and potent PPAR $\alpha / \gamma$-dual agonist (PAR5359) in two pre-clinical murine models, i.e., Citrobacter rodentium-induced infectious colitis and DSS-induced colitis. Mechanistically, we show that such balanced dual agonism promotes bacterial clearance more efficiently than individual agonists both in vivo and in vitro; PPAR $\alpha$ is required and its agonism is sufficient to induce the pro-inflammatory cytokines and cellular ROS, which are essential for bacterial clearance and immunity, whereas PPAR $\gamma$-agonism blunts these responses, delays microbial clearance and induces the anti-inflammatory cytokine, IL10. Balanced agonism achieved controlled inflammation while protecting the gut barrier and 'reversal' of the transcriptomic network. Furthermore, dual agonism effectively reversed the defective bacterial clearance observed in PBMCs derived from IBD patients. These findings not only deliver a macrophage modulator for use as barrier-protective therapy in IBD, but also highlight the potential of BoNE to accelerate and enhance the precision of drug discovery.

\section{KEY WORDS:}

Boolean Implication Network,

Inflammatory Bowel Disease (IBD),

80 Reactive Oxygen Species (ROS),

81 Macrophage,

82 Bacterial clearance,

83 Citrobacter,

84 PPAR $\alpha / \gamma$-dual agonists

$85 \quad$ PAR5359 
Inflammatory bowel disease (IBD) is an autoimmune disorder of the gut in which diverse components including microbes, genetics, environment and immune cells interact in elusive ways to culminate in overt diseases ${ }^{1-3}$. It is also heterogeneous with complex sub-disease phenotypes (i.e., strictures, fistula, abscesses and colitis-associated cancers) ${ }^{4,5}$. Currently, patients are offered anti-inflammatory agents that have a $\sim 30-40 \%$ response-rate, and $40 \%$ of responders become refractory to treatment within one year ${ }^{6,7}$. Little is known to fundamentally tackle the most widely recognized indicator/predictor of disease relapse i.e., a compromised mucosal barrier. Homeostasis within this mucosal barrier is maintained by our innate immune system, and either too little or too much reactivity to invasive commensal or pathogenic bacteria, is associated with $\mathrm{IBD}^{8}$. Although defects in the resolution of intestinal inflammation have been attributed to altered monocyte-macrophage processes in IBD, macrophage modulators are yet to emerge as treatment modalities in IBD $^{8}$.

We recently developed and validated an AI-guided drug discovery pipeline that uses large transcriptomic datasets (of the human colon) to build a Boolean network of gene clusters 9; this network differs from other computational methods (e.g., Bayesian and Differential

103 Expression Analyses) because gene clusters here are interconnected by directed edges that represent Boolean Implication Relationships that invariably hold true in every dataset within the cohort. Once built, the network is queried using machine learning approaches to identify in an unbiased manner which clusters most effectively distinguish healthy from diseased samples and do so reproducibly across multiple other cohorts (906 human samples, 234 mouse samples). Gene-clusters that maintain the integrity of the mucosal barrier emerged as the genes that are invariably downregulated in IBD, whose pharmacologic augmentation/induction was predicted to 'reset' the network. These insights were exploited to prioritize one target, choose appropriate pre-clinical murine models for target validation and design patient-derived

112 organoid models. Treatment efficacy was confirmed in patient-derived organoids using 113 multivariate analyses. This AI-assisted approach provided a first-in-class epithelial barrier114 protective agent in IBD and predicted Phase-III success with higher accuracy over traditional 115 approaches ${ }^{9}$.

116 Here we use the same AI-guided drug discovery pipeline, this time to identify and 117 validate a first-in-class macrophage modulator that is predicted to restore mucosal barrier and 118 homeostasis in IBD. We demonstrate the accuracy and predictive power of this networkrationalized approach and reveal the efficacy of balanced dual agonists of PPAR $\alpha / \gamma$ in two pre- 
clinical murine models and in patient-derived PBMCs. We also reveal the mechanism(s) of action that enable balanced agonists of this pair of nuclear receptors to reverse some of the fundamental imbalances of the innate immune system in IBD, such that immunity can be achieved without overzealous inflammation.

\section{RESULTS}

\section{Development of a web-based platform for generating a 'target report card'}

We first developed an interactive, user-friendly web-based platform that allows the querying of our Boolean network-based-IBD map ${ }^{9}$ with just a few intuitive clicks of the mouse, even for biologists (Supplementary Fig. 1). With the goal of enabling researchers to pick highvalue targets, we programmed the platform to generate a comprehensive automated 'target report card' that contains predictions on five components (Fig. 1A): (i) Impact on the outcome of IBD in response to treatment, which shows how levels of expression of any proposed target gene(s) relates to the likelihood of response to therapies across diverse cohorts; (ii) Therapeutic index, a computationally generated index using Boolean implication statistics which provides a likelihood score of indicate whether pharmacologic manipulation of the target gene(s) would lead to success in Phase III clinical trials; (iii) Appropriateness of mouse model, a component that indicates which murine models of colitis shows the most significant change in the target genes (and hence, likely to be best models to test the efficacy of any manipulation of that target); (iv) Gender bias, a component that indicates whether the gene is differentially expressed in IBD-afflicted men versus women; and (v) Target tissue/cell type specificity, which shows the likely cell type where the target is maximally expressed, and hence, the cell type of desirable pharmacologic action. Details of how therapeutic index is computed are outlined in Methods and in Supplementary Fig. 2; it is essentially a statistical score of how tightly any proposed target gene(s) associates with FDA-approved targets versus those that failed. Similarly, details of how cell type of action is computer are outlined in Methods and in Supplementary Fig. 3.

151 Previous work had identified a little over 900 genes in 3 clusters (Clusters \#1-2-3 within the

152 IBD map; Supplementary Fig. 1A-B) as potentially high-value targets, all of which were invariably downregulated in IBD-afflicted colons ${ }^{9}$. Reactome analyses showed that epithelial 
tight junctions (TJs), bioenergetics, and nuclear receptor pathway (PPAR signaling) related genes that are responsible for colon homeostasis are the major cellular processes regulated by these genes (Supplementary Fig. 1B). Downregulation of genes in clusters \#1-3 was invariably associated also with upregulation of genes in clusters \#4-5-6; reactome analyses of the latter showed cellular processes that concern immune cell activation, inflammation and fibrosis, which are hallmarks of IBD (Supplementary Fig. 1B). Of the druggable candidates within C\#1-2-3, 17 targets were identified as associated with GO biological function of 'response to stress'/'response to stimuli'. Targeting one of the 17 targets, PRKAB1, the subunit of the heterotrimeric AMP-kinase engaged in cellular bioenergetics and stress response successfully restored the gut barrier function and also protected it from collapse in response to microbial challenge ${ }^{9}$. Here, we prioritized two more of those 17 targets, PPARA and PPARG, which encode a pair of nuclear receptors, PPAR $\alpha$ and PPAR $\gamma$. These two stress/stimuliresponsive genes are equivalent to each other and to PRKAB1, and like PRKAB1, are invariably downregulated in all IBD samples (Supplementary Fig. 1B-D). PPARA is in cluster \#2 and PPARG is in cluster \#3 (Supplementary Fig. 1B). They both were located on the two major Boolean paths associated with epithelial barrier and inflammation/fibrosis (Supplementary Fig. 1B) ${ }^{9}$. Together, these findings imply three things: (i) that PPARA/G are simultaneously downregulated in IBD, (ii) that such downregulation is invariably associated with inflammation, fibrosis and disruption of the epithelial barrier, and (iii) that simultaneous upregulation of PPARA/G with agonists may restore the gut barrier. The last point is particularly important because PPAR $\alpha / \gamma$ agonists are known to augment the expression of PPARA/G, and depletion of either reduced the expression of the other ${ }^{10}$.

Noteworthy, while the role of PPAR $\gamma$ in colitis has been investigated through numerous studies over the past 3 decades ${ }^{11-13}$ (Table 1), the role of PPAR $\alpha$ has been contradictory (Table 2), and their dual agonism has never been explored. PPAR $\gamma$ agonists undeniably ameliorate DSS-induced colitis ${ }^{13-15}$. Although claimed to be effective on diverse cell types in the gut (epithelium, T-cells, and macrophages), the most notable target cells of PPAR $\gamma$ agonists are macrophages and dendritic cells $\begin{array}{llllll}16 & 17 & 18 & 19\end{array}$. Furthermore, Phase I and II clinical trials with PPAR $\gamma$ agonists either alone ${ }^{20,21}$ or in combination with mesalamine ${ }^{22}$ show barrier protective effects in UC patients. Despite these insights, the biopharmaceutical industry has not been able to harness the beneficial impact of this major target within emergent therapeutic strategies largely due to a trail of withdrawals after devastating long-term side effects including heart failure, bone fracture, bladder cancer, fluid retention and weight gain ${ }^{23,24}$. Intriguingly, and of 
relevance to this work, the addition of PPAR $\alpha$-agonistic activity to PPAR $\gamma$ or $\delta$ agonists have led to a higher safety profile, leading to their development for use in many diseases, including type 2 diabetes, dyslipidemia and non-alcoholic fatty liver disease ${ }^{25}$.

\section{An automated target 'report card' for PPARA/G in IBD}

We next generated an automated target report card for PPARA/G. High levels of both PPARs were sufficient to distinguish healthy from IBD samples, not just in the test cohort that was used to build the IBD-map (ROC AUC of 0.74; Fig 1B; see also Supplementary Fig. 2A-D), but also in four other independent cohorts with ROC AUC consistently above 0.88 (Fig 1C). High levels of both PPARs also separated responders from non-responders receiving TNF $\alpha$ neutralizing mAbs, GSE16879, E-MTAB-7604 or Vedolizumab that block the $\alpha 4 \beta 7$ integrin to prevent selective gut inflammatory, GSE73661 (ROC AUC 0.63-0.89, Fig 1D), inactive disease from active disease (two independent cohorts ROC AUC above 0.93; Fig 1D), and quiescent UC that progressed, or not to neoplasia (ROC AUC $=1.00$ for qUC vs. nUC; Fig 1D). High level of PPARA/G was also able to distinguish healthy from diseased samples in diverse murine models of colitis (Fig 1E); but such separation was most effectively noted in some models (Citrobacter infection-induced colitis, adoptive T-cell transfer, TNBS and $I L 10^{-/-}$), but not in others (DSS, and TNFR $1 / 2^{-/-}$). These findings imply that therapeutics targeting these two genes are best evaluated in the murine models that show the most consistent decrease in the gene expression, e.g., Citrobacter infection-induced colitis, adoptive T-cell transfer, TNBS, etc. This was intriguing because the majority ( 90\%) of the published work on PPARA/G agonists have been carried out in DSS models (Table 1-2).

The expression profile of the target genes in the gut mucosa revealed that PPARA/G are co-expressed at the highest levels in the crypt top epithelial cells and macrophages (Fig 1F; Supplementary Fig. 3), predicting that dual agonists are likely to preferentially act on these two cell types. The therapeutic index was below 0.1 for both genes ( 0.06 for PPARA and 0.04 for PPARG; Fig 1G; Supplementary Fig. 2E-F), aligned well with two other FDA-approved targets shown on the line graph (ITGB1, 0.046 and JAK2, 0.032). The index, which is a statistical measure of the strength of association of PPARA/G with genes that are targets of FDA-approved drugs that have successfully moved through the three phases of drug discovery (i.e., proven efficacy, with acceptable toxicity). A low number is indicative of a high likelihood of success in Phase-III trials. Finally, PPARA/G expression was downregulated to a similar 
extent in both genders (Fig 1H), predicting that therapeutics targeting them are likely to be effective in both men and women.

\section{Rationalization of PPARA/G as targets in IBD}

Because proteins, but not transcripts, are the targets of therapeutic agents, the impact of therapeutics is translated to cellular processes via protein-protein interaction (PPI) networks, a.k.a interactomes. We next asked how dual agonists of PPAR $\alpha / \gamma$ might impact cellular pathways and processes. A PPI network visualized using PPAR $\alpha / \gamma$ as 'query/input' and the interactive STRING v11.0 database (https://string-db.org/) as a web resource of known and predicted protein-protein interactions curated from numerous sources, including experimental data, computational prediction methods and public text collections. PGC1a (a product of the gene PPARGC1A) was a common interactor between the two PPARs (Fig 2A). We noted that PGC1a also happens to be a major component within the PPAR $\alpha / \gamma$ functional network, serving as a central hub for positive feedback loops between the PPARs and their biological function (Fig 2B), i.e., mitochondrial biogenesis, DNA replication and energetics (electron transport chain and oxidative phosphorylation). When we analyzed the functional role of the interactomes of PPAR $\alpha / \gamma$ we noted that indeed both interactomes converged on lipid metabolism, mitochondrial bioenergetics and circadian processes (Fig 2C). These findings are consistent with the finding that PPARA/G and PPARGC1A are located within clusters \#1-2-3 and all of them are predicted to be progressively and simultaneously downregulated in IBD samples (Fig 2D; based on the IBD map, Supplementary Fig. 1).

\section{PPARA/G is downregulated in Ulcerative colitis and Crohn's Disease}

Previous work demonstrated that both PPAR $\alpha$ and PPAR $\gamma$ are highly expressed in the colon ${ }^{26}$. They have also shown that both PPAR $\alpha / \gamma$ protein and mRNA are downregulated (by $\sim 60 \%$ ) in active UC and the expression of PPAR $\gamma$ was significantly associated with disease activity ${ }^{27}$. This impaired expression was found in both inflamed and noninflamed areas ${ }^{28}$. Polymorphisms have also been detected in PPAR $\gamma$; while some studies found those to be associated with an increased risk for $\mathrm{CD}^{29,30}$, others found no evidence suggesting any form of association with an increased disease risk ${ }^{31}$. We collected endoscopically obtained biopsies from the colons of healthy $(n=7)$ and IBD $(n=14$ and 14 of UC and CD, respectively) patients and assessed the levels of transcripts for PPARA/G and PPARGC1A by qPCR (Fig 2E). We confirmed that all three transcripts were significantly downregulated in UC and CD samples 
compared to healthy; both PPARG and PPARGC1a were more significantly downregulated in

253 CD compared to UC (Fig 2F). These findings are in keeping with the network-based 254 predictions that these genes should be downregulated invariably in all IBD samples, regardless of disease subtype (see individual disease maps; Supplementary Fig. 4-5). While both PPARA and PPARG are in cluster \#2 in the UC map, PPARG and PPARA are in separate clusters, clusters 2 and 6, respectively, in the CD map (Supplementary Fig. 4-5). Reactome pathway analyses implied that in the case of UC, the two nuclear receptors may co-regulate similar cellular homeostatic processes associated with cluster \#2, i.e., mitochondrial biogenesis and translation initiation, infectious disease and detoxification of ROS (see Supplementary Fig. 4). By contrast, in the case of $\mathrm{CD}$, they may independently regulate diverse cellular processes that maintain cellular homeostasis; while PPARG is associated with cellular metabolism (TCA cycle) and inhibition of NFkB signaling, PPARA is associated with transcriptional activity of nuclear receptors, cholesterol biosynthesis and Met/Ras signaling (see Supplementary Fig. 5). Taken together, these findings demonstrate that both targets are downregulated in IBD and that they may regulate key pathophysiologic processes that are vital for cellular homeostasis.

\section{Synthesis and validation of PAR5359, a potent and specific PPAR $\alpha / \gamma$ dual agonist}

We noted that all commercially available PPAR $\alpha / \gamma$ dual agonists lack 'balanced' agonistic activities (Table 3) ${ }^{32,33}$. Drugs that have fallen aside due to safety concerns also lack balanced agonism; most of them are more potent on PPAR $\gamma$ than on PPAR $\alpha$ by a log-fold (Table 3). All these PPAR $\alpha / \gamma$ dual agonists have been withdrawn due to safety concerns ${ }^{25}$, but the cause of the 'unsafe' profile remains poorly understood. Saroglitazar, the drug that is the only active ongoing Phase-III trial (NCT03061721) in this class, has $\sim 3$ log-fold more potency on PPAR $\alpha$ than PPAR $\gamma^{34}$. Because our AI-guided approach suggested the use of simultaneous and balanced agonism, we favored the use of the only balanced and yet, specific PPAR $\alpha / \gamma$ agonist described to date, PAR5359 ${ }^{35,36}$ (see Table 4). In the absence of commercial sources or welldefined methods on how to synthesize this molecule, we generated PAR5359 in 4 synthetic steps (see details in Methods) and confirmed its specificity and the comparable agonistic activities using pure single PPAR $\alpha\left[\mathrm{GW} 764{ }^{37}\right]$ or PPAR $\gamma\left[\right.$ Pioglitazone $\left.{ }^{38}\right]$ agonists as controls (Supplementary Fig. 6). With these potent and specific compounds as tools, and their doses adjusted to achieve the same potency, we set out to validate the network-based predictions using pre-clinical models. 
We next sought to assess the efficacy of individual and dual agonists of our compounds in murine pre-clinical models. PPAR $\alpha / \gamma$ 's role (or the role of their agonists) in protecting the gut barrier has been evaluated primarily in DSS-induced colitis (Table 1, 2). However, BoNE prioritized other models over DSS, many of which accurately recapitulate the PPAR $\alpha / \gamma$-downregulation that is observed in the barrier-defect transcript signature in human IBD (Fig. 1E). Among those, we chose $C$. rodentium-induced infectious colitis, a robust model to study mucosal immune responses in the gut and understand derailed host-pathogen interaction in IBD ${ }^{39-41}$. Furthermore, this model requires the balanced action of macrophages (a cell line predicted to be the preferred cell type target; Fig. 1F) to promote bacterial clearance and healing ${ }^{42}$.

Colitis was induced by oral gavage of $C$. rodentium and mice were treated daily with the drugs via the intraperitoneal route (see workflow detailed in Fig. 3A, Supplementary Fig. 7A). The dose for each drug was chosen based on their $E C_{50}$ on their respective targets so as to achieve equipotent agonistic activities (Supplementary Fig. 6). Fecal pellets of individual mice were collected to determine the number of live bacteria present in the stool. As anticipated, the bacterial burden in all mice increased from day 5 , reaching a peak on day 7 , forming a plateau until day 11 before returning to pre-infection baseline by day 18 (Fig. 3B). Compared against all other conditions, PAR5359-treated mice cleared the gut bacterial load significantly and rapidly (Fig. 3B-D). Citrobacter infection was associated with significant epithelial damage and profuse infiltration of inflammatory cells and edema by day 7 (Supplementary Fig. 7B) most of which resolved by day 18 (DMSO control; Fig. 3E). Colons collected on day 7 showed that treatment with PAR5359 significantly reduced these findings when compared to vehicle (DMSO), PPAR $\alpha$ and PPAR $\gamma$ agonists alone (Supplementary Fig. 7B). Unexpectedly, when we analyzed the colons on day 18, we noted persistent immune infiltrates in tissues in two treatment arms, pioglitazone and GW7647 (arrowheads; Fig. 3E), but not in the vehicle control group, or those treated with PAR5359. These findings indicate that individual PPAR $\alpha$ or PPAR $\gamma$ agonists may either retard bacterial clearance and/or induce an overzealous amount of inflammation, but the balanced dual agonist (PAR5359) may have

314 effectively cleared infection and resolved inflammation. PAR5359 also reduced spleen 315 inflammation as evidenced by a decreased spleen weight and length compared to vehicle 316 control (Supplementary Fig. 7C-F). The spleens of mice treated with DMSO, PPAR $\alpha$-alone 317 agonist, GW7647 and PPAR $\gamma$-alone agonist, Pioglitazone showed black-discoloration, 
318 presumably infarcts (arrows, Supplementary Fig. 7C, 7E). Notably, the spleens of mice 319 treated with PPAR $\alpha$-alone agonist, GW7647, showed a significant increase in spleen length 320 (Supplementary Fig. 7D, 7F).

321 Taken together, these findings indicate that PPAR $\alpha / \gamma$ dual agonist PAR5359 is superior in ameliorating $C$. rodentium-induced colitis than either PPAR $\alpha$ or PPAR $\gamma$ agonist used alone. Treatment with the dual, but not the single agonists hastened bacterial clearance, resolved inflammation, and induced healing.

\section{PAR5359 resists $C$. rodentium-induced gene expression changes in the colon}

We next sought to determine if pharmacologic augmentation of PPARA/G was sufficient to influence the entire transcriptomic network via the invariant Boolean implication relationships between the gene clusters. RNA sequencing (RNA-seq) studies were carried out on the $C$. rodentium-infected colons in each treatment group (Fig. 3A). As expected, downregulation of genes in clusters \#1-2-3 of the IBD-map was significant in infected (DMSO) vs uninfected controls, indicative of network shift from health towards disease (Fig. 3F); PAR5359-treatment resisted such shift.

Pre-ranked gene set enrichment analyses (GSEA) based on pair-wise differential expression analysis showed that when compared to DMSO control, dual PPAR $\alpha / \gamma$ agonism with PAR5359, but not individual agonists Pioglitazone or GW7647 was able to significantly preserve epithelial junction signatures (both tight and adherens junctions) and balance macrophage processes (compare Fig. 3G with Supplementary Fig. 8A). These findings are in keeping with the predictions that epithelial cells and macrophages maybe the primary cell type of action for dual PPAR $\alpha / \gamma$ agonists. Comparison of all treatment cohorts against each other revealed that although both PAR5359 and Pioglitazone were superior to GW7647 in maintaining some epithelial processes (differentiation, tight junctions) and macrophage processes (Supplementary Fig. 8B-E), PAR5359 emerged as the only group that maintained homeostatic PPAR signaling in nature and extent as uninfected control (Supplementary Fig. 8F).

Taken together, these findings suggest that dual agonists of PPAR $\alpha / \gamma$ are sufficient to either resist network shift and/or reverse the disease network in the setting of colitis. They also offer clues suggestive of epithelial and macrophage processes, two key cellular components of innate immunity in the gut lining as major mechanisms. These transcriptome wide impacts 
suggest that PPAR $\alpha / \gamma$ dual agonist PAR5359 is superior in restoring colon homeostasis in $C$. rodentium-induced colitis than either PPAR $\alpha$ or PPAR $\gamma$ agonist used alone.

\section{PAR5359 ameliorates DSS-induced colitis}

354 It is well known that no single mouse model recapitulates all the multifaceted complexities of IBD $^{43,44}$. Because almost all studies evaluating PPAR $\alpha / \gamma$-modulators have been performed on the DSS-induced colitis model (Table 1-2), we asked whether the PPAR $\alpha / \gamma$ dual agonist PAR5359 can ameliorate colitis in this model. Mice receive intrarectal DMSO vehicle control or PAR5359 while receiving DSS in their drinking water (Supplementary Fig. 9A). Disease severity parameters, i.e., weight loss, disease activity index, shortening of the colon and histology score were significantly ameliorated in the PAR5359-treated group (Supplementary Fig. 9A-E). These findings show that the PPAR $\alpha / \gamma$-dual agonist, PAR5359, is also effective in DSSinduced colitis. It is noteworthy that the PAR5359 dual agonist offered protection in the DSSmodel, because prior studies using the same model have demonstrated that PPAR $\alpha$-agonists worsen ${ }^{45,46}$, and that the PPAR $\gamma$-agonists ameliorate colitis ${ }^{47-49}$ (see Table 1-2).

PAR5359 promotes bacterial clearance with controlled production of ROS and

\section{inflammation in peritoneal macrophages}

Because the intestinal macrophages play a crucial role in maintaining the integrity of the gut epithelial barrier and in the control of pathogen invasion by triggering an appropriate immune response, the fine-tuning macrophage is essential in maintaining homeostasis and, potentially, the development of IBD. To explore the mechanism(s) by which dual, but not individual, PPAR agonists may alter macrophage response to microbes, we incubated macrophages treated or not with the drugs and challenged them with $\mathrm{CD}$-associated adherent invasive E. coli (AIEC)$L F 82$; this strain, originally isolated from a chronic ileal lesion from a CD patient ${ }^{50}$. As for the source of macrophages, we isolated metabolically active primary murine peritoneal macrophages using Brewer thioglycolate medium using established protocols ${ }^{51,52}$. These macrophages are known to have high phagocytic activity ${ }^{51}$ (Fig. 4A). Thioglycolate-induced peritoneal macrophages (TG-PMs) were lysed, and viable intracellular bacteria were counted after plating on an agar plate. Pre-treatment with $1 \mu \mathrm{M}$ PAR5359 and an equipotent amount of GW7647 (PPAR $\alpha$ agonist) promoted bacterial clearance and reduced the bacterial burden when compared to vehicle control (Fig. 4B). By contrast, pre-treatment with Pioglitazone 
higher at both $3 \mathrm{~h}$ and $6 \mathrm{~h}$ after infection (Fig. 4B). Reduced clearance of microbes in the latter was associated also with reduced cellular levels of reactive oxygen species (ROS) (Fig. 4C); oxidative burst and induction of ROS is key component for effective bacterial killing 53,54 . PAR5359 did not interfere with the production of microbe-induced ROS, and the PPAR $\alpha$ agonist (GW7647) was permissive to ROS induction (in fact, even induces it over bacteriaalone control) during initial time points after infection (Fig. 4C).

These patterns of microbial clearance and cellular ROS were associated also with the expression of cytokines, as determined by qRT-PCR analyses (Fig. 4D). As expected, infection of TG-PM with AIEC-LF82 induced ill $\beta$, il6, tnf $\alpha$ and illo. PAR5359 significantly and selectively suppressed the expression of the pro-inflammatory cytokines ill $\beta$, il6 and tnf $\alpha$ (but not the anti-inflammatory cytokine, il10) (Fig. 4D). By contrast, the PPAR $\gamma$-specific agonist pioglitazone significantly and indiscriminately suppressed all the cytokines, while there was no effect of the PPAR $\alpha$ specific agonist GW7647 (Fig. 4D). ELISA studied on the supernatant media further confirmed these findings (Fig. 4E), demonstrating that the effects in gene expression were also translated to the levels of secreted cytokine protein released by the macrophages in the supernatant.

It is noteworthy that for the most part, the qPCR (Fig. 4D) and ELISA (Fig. 4E) studies matched, except illo; although pioglitazone appeared to suppress illo mRNA, it did not suppress the levels of the illo protein, suggesting that PPAR $\gamma$-agonist is sufficient for an overall anti-inflammatory phenotype. Similarly, although GW7647 appeared to not affect $i l 10$ mRNA, it suppressed the levels of the illo protein, suggesting that PPAR $\alpha$ agonist is sufficient for an overall pro-inflammatory phenotype. Similar findings were also observed in the case of another enteric pathogen, S. enterica, i.e., unlike the dual agonist, neither PPAR $\alpha$ nor PPAR $\gamma$ agonist could enhance bacterial clearance with a modest induction of pro-inflammatory cytokines (significantly lower than control) and, yet, had no impact on anti-inflammatory IL10 production (Supplementary Fig. 10).

Taken together, these results show that- (i) PPAR $\gamma$-agonism induces 'tolerance' by suppressing inflammation, inhibiting ROS production and delaying bacterial clearance; (ii) PPAR $\alpha$-agonism enhances the induction of inflammation and ROS, and promotes bacterial clearance; and (iii) PPAR $\alpha / \gamma$-dual agonism strikes a somewhat balanced response. The latter suppresses proinflammatory cytokines without suppressing anti-inflammatory cytokine il10, and is permissive to inflammation and ROS induction that is optimal and sufficient to promote bacterial clearance. 
417 To further dissect which nuclear receptors are responsible for the balanced actions of the dual

418 agonist, we next used a set of highly specific and potent PPAR $\alpha / \gamma$-inhibitors (Table 4). We pre-treated TG-PMs with PPAR $\alpha$ and PPAR $\gamma$ inhibitors, either alone, or in combination, followed by stimulation with bacterial cell wall component LPS (Fig. 5A). As expected, LPS induced the cellular levels of ROS (Fig. 5B) and inflammatory cytokines (Fig. 5C-D) in TGPMs significantly higher than in untreated control cells. Inhibition of PPAR $\alpha$ suppressed the induction of cellular ROS and inflammatory cytokines, both at the level of gene and protein levels (Fig. 5B-D). By contrast, inhibition of PPAR $\gamma$ did not interfere with either response (Fig. 5B-D). Simultaneous inhibition of both PPAR $\alpha$ and PPAR $\gamma$ mimicked the cellular phenotypes in the presence of PPAR $\alpha$-inhibitors (Fig. 5B-D), indicating that inhibition of PPAR $\alpha$ is sufficient to recapitulate the phenotype of dual inhibition. Taken together, these findings indicate that PPAR $\alpha$ is required for the proinflammatory response of macrophages.

PPAR $\alpha / \gamma$ dual agonist PAR5359 promotes bacterial clearance in patient-derived PBMCs In search of a pre-clinical human model for testing drug efficacy, we next assessed microbial handling by PBMCs derived from patients with IBD and compared them with that in agematched healthy volunteers. We enrolled both male and female patients and both $\mathrm{CD}$ and UC (Table 5). Consecutive patients presenting for routine care to the UC San Diego IBD clinic were enrolled into the study; the only exclusion criteria were failure to obtain informed consent for the study or active infections and/or disease flare. Peripheral blood collected in the clinic was freshly processed as outlined in Fig. 6A to isolate PBMCs. Pre-treatment for 30 min with vehicle or PAR5359 was followed by infection for 1h. Subsequently, the cells were treated with gentamicin for $60 \mathrm{~min}$ to kill extracellular bacteria to assess intracellular bacterial burden at 1 and $6 \mathrm{~h}$ after the gentamicin wash.

Two observations were made: First, CD but not UC patient-derived PBMCs when infected with $A I E C$-LF82 showed an increased number of internalized viable bacteria when compared to healthy PBMCs (Fig. 6B, 6E), indicative of either defective clearance and/or increased permissiveness to bacterial replication within the cells is limited to the CD. Second, pre-treatment with PAR5359 could improve clearance significantly (Fig. 6C-D, 6F-G). These results indicate that bacterial clearance is delayed in PBMCs of patients with $\mathrm{CD}$ and that PPAR $\alpha / \gamma$ dual agonism with PAR5359 can reverse that defect. The possibility that such 
out (see bacterial viability assay in Supplemental Fig. 11). Our findings demonstrate that bacterial clearance is delayed primarily in $\mathrm{CD}$ and not $\mathrm{UC}$ are in keeping with the fact that delayed bacterial clearance from inflamed tissues (up to $\sim 4$-fold) is uniquely observed in CD 55. These findings are also in keeping with our own observation that the downregulation of PPARG/PPARGC1A was more prominent in patients with CD (Fig. 2E-F). In fact, delayed clearance is one of the major reasons for persistent inflammation and disease progression among patients with $\mathrm{CD}^{55,56}$.

\section{DISCUSSION}

458 Barrier-protection/restoration is the treatment endpoint for all clinical trials in IBD therapeutics; however, despite much success in the development of anti-inflammatory therapies ${ }^{7,57}$, barrier-protective therapeutics in IBD have been slow to emerge ${ }^{58}$. Here we report the discovery of an effective barrier-protective therapeutic strategy in IBD identified using an AI-guided navigation framework (summarized in Fig 7). First, a network-based drug discovery approach ${ }^{9}$ was used to identify, rationalize and validate dual and balanced agonism of PPAR $\alpha / \gamma$ (but not one at a time) is necessary for therapeutic success. Second, we provided evidence in the form of proof-of-concept studies (in two different pre-clinical murine models) demonstrating that the simultaneous and balanced agonistic activation of the pair of PPARs as an effective barrier protective strategy in IBD. Third, we demonstrate that macrophages are one of the primary target cell type of this therapeutic strategy; dual agonist (but not single) was permissive to the induction of macrophage responses expected for optimal immunity without overzealous inflammation. There are three notable takeaways from this study, which are unexpected observations and/or insights that fill key knowledge gaps in the fields of - (a) network medicine, (b) IBD therapeutics and (c) macrophage biology.

First, with regard to network medicine, the AI-guided approach we used here differs from the current practice in three fundamental ways: 1) Unlike most studies that prioritize targets based on Differential Expression Analysis (DEA, or integrated DEA) or Bayesian approaches, target identification and prediction, this work was guided by a Boolean implication network of continuum states in human disease ${ }^{9}$;2) Instead of conventional approaches of trialand-error, intuitive guess and/or knowledge-based prioritization of study models (animal or cell-type of action), target validation in network-rationalized animal and cell-type models that most accurately recapitulate the role of the target(s) during disease progression; 3) Inclusion of human pre-clinical model (patient-derived PBMCs) for target validation, inspiring the concept of Phase '0' trials that have the potential to personalize the choice of therapies. The combined 
synergy of these approaches validates a first-in-class macrophage modulator in addressing the broken gut barrier in IBD.

The impact of using such an approach is 4-fold: (i) Because the network approach used here relies on the fundamental invariant Boolean implication relationships between genes, and their patterns of changes in expression between healthy and IBD samples, such 'rule of invariant' implies that any given relationship and/or change in expression pattern annotated within the network must be fulfilled in every IBD patient. By that token, targets/drugs prioritized based on this network is expected to retain efficacy beyond inbred laboratory mice, into the heterogeneous patient cohorts in the clinic. (ii) This AI-guided approach not just helped compute pre-test probabilities of success ("Therapeutic Index"), but also helped pick models that are most insightful and appropriate to demonstrate therapeutic efficacy (e.g., Citrobacter rodentium infection-induced colitis) and to pinpoint the cell type and mechanism of action (microbial clearance by macrophages). This is noteworthy because the conventional approach in studying PPARs has been limited to the use of DSS-induced colitis (see Table 1-2), which has often given conflicting results (see Table 2). In fact, without the use of the Citrobacter rodentium infectious colitis model, the deleterious effects of PPAR $\gamma$ agonists would have been overlooked. (iii) Having a computational framework improves precision in target identification; it is because of the emergence of the two PPARs (alongside their positive feedback regulator, PGC1a) within our network, we rationalized their dual agonism as a preferred strategy (over single) and our experiments validated that prediction both in vivo and in vitro. This is noteworthy because conventional approaches have demonstrated a protective role of PPAR $\gamma$ agonists and a conflicting (both protective and exacerbating) role of PPAR $\alpha$ in IBD ${ }^{46,59-61}$; the advantage of dual agonism has neither been rationalized nor tested. (iv) The 'target report card', like the one shown here, is a project navigation tool that is geared to streamline decision-making (i.e., which genes, which animal models, which cell type/cellular process, what is the likelihood of success, etc.), which in turn should reduce attrition rates, waste and delays; the latter are well-recognized flaws in the current process of drug discovery. combinations of PPAR agonists are inferior to dual/balanced agonists. Conventional and reductionist approaches have inspired numerous studies with single PPAR agonists over the past decade (Tables 1-2). However, given the devastating side effects of most single or unbalanced PPAR $\alpha / \gamma$ agonists (Table 3), translating to the clinic beyond a Phase II trial 20,62,63

515 has not been realized. Because the therapeutic index for the dual PPAR $\alpha / \gamma$ agonists matches 
that of other FDA-approved targets/drugs, it is predicted that barring unexpected side effects, dual PPAR agonists are likely to be effective as barrier-protective agents. As for side effects,

518 we noted is that balanced PPAR $\gamma / \alpha$ agonists are rare; while all dual PPAR $\alpha / \gamma$ agonists that have 519 been discontinued due to side effects happen to be either single (only PPAR $\gamma$ ) or 'unbalanced' 520 (PPAR $\gamma>>$ PPAR $\alpha$ agonistic activity), the newer generation formulations that are currently in 521 the clinical trial have a reversed agonistic potency (PPAR $\alpha>>$ PPAR $\gamma$ agonistic activity) (see 522 Table 3). Because macrophage responses require finetuning (discussed below), our studies 523 show how unopposed agonism of either PPAR $\gamma$ or PPAR $\alpha$ is harmful and can impair/dysregulate the way macrophages respond when microbes breach past the gut barrier. It is possible that many of the side effects of the discontinued thiazolidinediones are due to their inability to achieve that 'optimal' spectrum of macrophage function.

Third, when it comes to macrophage biology, this work sheds some unexpected and previously unforeseen insights into the role of the PPARs in the regulation of macrophage processes. Extensively studied for over $\sim 3$ decades, PPARs are known to regulate macrophage activation in health and disease ${ }^{64}$. Targeting PPARs as a host-directed treatment approach to infectious/inflammatory diseases appears to be a sound strategy because they regulate macrophage lipid metabolism, cholesterol efflux, inflammatory responses (ROS and cytokine production), apoptosis, and production of antimicrobial byproducts ${ }^{65}$. We found that unopposed PPAR $\gamma$ activation suppresses bacterial clearance and blunts the induction of proinflammatory (but not anti-inflammatory, IL10) cytokines and ROS in response to infection both in vivo and in vitro. In other words, and consistent with prior reports, PPAR $\gamma$ activation suppressed inflammation at the cost of impairing immunity. Our findings are in keeping with the findings of a systematic review and meta-analysis of 13 long-term randomized controlled trials that involved 17,627 participants $(8,163$ receiving PPAR $\gamma$ agonists and 9,464 receiving control drugs $)^{66}$. Long-term $(\sim 1-5.5 \mathrm{y})$ use of PPAR $\gamma$ agonists increases the risk of pneumonia or lower respiratory tract infection significantly, some of which result in hospitalization, disability, or death ${ }^{66}$. In the case of PPAR $\alpha$, unopposed activation-induced ROS and proinflammatory cytokines and accelerated bacterial clearance. Inhibitor studies further confirmed that PPAR $\alpha$ was required for these responses (Fig 5). These findings are in keeping with others' showing that PPAR $\alpha$, but not PPAR $\gamma$ is required for NADPH-induced ROS formation both in human and murine macrophages ${ }^{67}$. PPAR $\alpha$ agonists induce the expression of NADPH oxidase subunits p47(phox), p67phox, and gp91phox, which are all essential functional components of NADPH complex ${ }^{67}$. Dual and balanced PPAR $\alpha / \gamma$ agonism enhanced 
bacterial clearance with only a moderate induction of proinflammatory cytokines or ROS. Such a response ensures that the macrophage functions within a 'goldilocks' zone, mounting inflammation that is just sufficient for microbial clearance and immunity. In our analysis, the only other PPAR-related gene within the IBD network, i.e., PGCla, and its role within the PPAR $\alpha / \gamma$ axis suggests that the intricate network of forward feedback loops orchestrated by PGCla may be critical for achieving the critical balance between immunity and inflammation, which is a key outcome of the dual PPAR $\alpha / \gamma$ agonists.

Because previous studies using cell-specific gene depletion have indicated that the barrier-protective role of PPAR $\gamma$ may be mediated via cells other than the macrophages ${ }^{48}$, namely, the T cells ${ }^{68}$ and the epithelial cells ${ }^{69}$, it is possible that the dual PPAR $\alpha / \gamma$ agonists also act on those cells, promoting bacterial clearance and balancing cellular bioenergetics, ROS and cytokine production, in manners similar to that we observe in macrophages.

Taken together, our study uses an unconventional approach to rationalize and validate the use of PPAR $\alpha / \gamma$ dual agonists as first-in-class barrier protective macrophage modulators in the management of IBD. The approach is powerful because it leverages the precision of mathematics (Boolean algebra of logic) and the fundamental invariant patterns in gene expression (Boolean Implications). The AI-navigated drug discovery approach defined here could serve as a blueprint for future studies not just in IBD, but in any other such complex chronic diseases.

\section{AUTHOR CONTRIBUTIONS}

\section{Role}

\section{Authors}

\section{Conceptualization; Supervision; Project administration}

- Conceptualization (PG and GDK)

- Supervision (PG, SD, DS)

PG, DS, SD and

- Project Administration (PG)

\section{Investigation; Methodology; Data curation, Formal Analysis}

GDK, VC, MSA, EV, IMS, DT, FU, JRS, SD

- Animal studies (GDK, VC, MSA, IMS, FU)

- Cell and tissue analysis (qPCR, ROS, bacterial clearance, ELISA)- (GDK, VC, MSA, IMS, DT, FU, SD)

- Computational modeling (DS)

- Computational analysis (DS)

\section{Funding acquisition}

\section{Resources}

\section{Software}

Visualization; Writing - original draft; Writing - review \& editing

DS, SD, PG

JRS, GL, JY, WJS

DS

GDK, DS, PG 
572 We thank Dharanidhar Dang (UCSD) for comments and critiques during the preparation of the manuscript. This work was supported by National Institutes for Health (NIH) grants R01AI141630 (to PG), DK107585 (to SD). PG, SD, and DS were also supported by the Leona M. and Harry B. Helmsley Charitable Trust and the NIH (UG3TR003355, UG3TR002968 and R01-AI55696). GDK was supported through The American Association of Immunologists Intersect Fellowship Program for Computational Scientists and Immunologists. J.S. acknowledges support from the Interfaces Training Grant at UCSD (NIH T32EB009380). Authors thank to Lee Swanson, Courtney Tindle, Stella-Rita Ibeawuchi, Julian Tam and Madhubanti Mullick for their comments, feedback and technical support. This manuscript includes data generated at the UC San Diego Institute of Genomic Medicine (IGC) using an Illumina NovaSeq 6000 that was purchased with funding from a National Institutes of Health SIG grant (\#S10 OD026929). Additionally, a P30 grant (NIH/NIDDK, P30DK120515) subsidized the RNA Seq and histology work showcased here.

\section{CODE AVAILABILITY}

The codes are publicly available at the following links: https://github.com/sahoo00/BoNE; https://github.com/sahoo00/Hegemon

\section{REFERENCES}

1 Richard, M. L. \& Sokol, H. The gut mycobiota: insights into analysis, environmental interactions and role in gastrointestinal diseases. Nat Rev Gastroenterol Hepatol 16, 331-345, doi:10.1038/s41575-019-0121-2 (2019).

2 Schirmer, M., Garner, A., Vlamakis, H. \& Xavier, R. J. Microbial genes and pathways in inflammatory bowel disease. Nat Rev Microbiol 17, 497-511, doi:10.1038/s41579019-0213-6 (2019).

3 Lavelle, A. \& Sokol, H. Gut microbiota-derived metabolites as key actors in inflammatory bowel disease. Nat Rev Gastroenterol Hepatol, doi:10.1038/s41575-0190258-z (2020).

4 Furey, T. S., Sethupathy, P. \& Sheikh, S. Z. Redefining the IBDs using genome-scale molecular phenotyping. Nat Rev Gastroenterol Hepatol 16, 296-311, doi:10.1038/s41575-019-0118-x (2019).

5 Olivera, P., Danese, S., Jay, N., Natoli, G. \& Peyrin-Biroulet, L. Big data in IBD: a look into the future. Nat Rev Gastroenterol Hepatol 16, 312-321, doi:10.1038/s41575019-0102-5 (2019).

6 Moschen, A. R., Tilg, H. \& Raine, T. IL-12, IL-23 and IL-17 in IBD: immunobiology and therapeutic targeting. Nat Rev Gastroenterol Hepatol 16, 185-196, doi:10.1038/s41575-018-0084-8 (2019).

7 Ahluwalia, J. P. Immunotherapy in inflammatory bowel disease. Med Clin North Am 
6118 Na, Y. R., Stakenborg, M., Seok, S. H. \& Matteoli, G. Macrophages in intestinal

612

613

614

615

616

617

618

619

620

621

622

623

624

625

626

627

628

629

630

631

632

633

634

635

636

637

638

639

640

641

642

643

644

645

646

647

648

649

650

651

652

653

654

655

656

657

658

659 inflammation and resolution: a potential therapeutic target in IBD. Nat Rev Gastroenterol Hepatol 16, 531-543, doi:10.1038/s41575-019-0172-4 (2019).

9 Debashis, S. et al. AI-guided Discovery of a Barrier-Protective Therapy in Inflammatory Bowel Disease. Nat Commun (under review 2020).

10 Kim, S. H. et al. MD001, a Novel Peroxisome Proliferator-activated Receptor $\alpha / \gamma$ Agonist, Improves Glucose and Lipid Metabolism. Sci Rep 9, 1656, doi:10.1038/s41598-018-38281-0 (2019).

11 Annese, V., Rogai, F., Settesoldi, A. \& Bagnoli, S. PPARgamma in Inflammatory Bowel Disease. PPAR Res 2012, 620839, doi:10.1155/2012/620839 (2012).

12 Auwerx, J. Nuclear receptors. I. PPAR gamma in the gastrointestinal tract: gain or pain? Am J Physiol Gastrointest Liver Physiol 282, G581-585, doi:10.1152/ajpgi.00508.2001 (2002).

13 Dubuquoy, L. et al. PPARgamma as a new therapeutic target in inflammatory bowel diseases. Gut 55, 1341-1349, doi:10.1136/gut.2006.093484 (2006).

14 Ramakers, J. D. et al. The PPARgamma agonist rosiglitazone impairs colonic inflammation in mice with experimental colitis. J Clin Immunol 27, 275-283, doi:10.1007/s10875-007-9074-2 (2007).

15 Vetuschi, A., Pompili, S., Gaudio, E., Latella, G. \& Sferra, R. PPAR-gamma with its anti-inflammatory and anti-fibrotic action could be an effective therapeutic target in IBD. Eur Rev Med Pharmacol Sci 22, 8839-8848, doi:10.26355/eurrev_201812_16652 (2018).

16 Xia, H. et al. Protectin DX increases survival in a mouse model of sepsis by ameliorating inflammation and modulating macrophage phenotype. Sci Rep 7, 99, doi:10.1038/s41598-017-00103-0 (2017).

17 Nagy, L., Szanto, A., Szatmari, I. \& Széles, L. Nuclear hormone receptors enable macrophages and dendritic cells to sense their lipid environment and shape their immune response. Physiol Rev 92, 739-789, doi:10.1152/physrev.00004.2011 (2012).

$18 \mathrm{Xu}, \mathrm{Z}$. et al. PPAR- $\gamma$ agonist ameliorates liver pathology accompanied by increasing regulatory B and T cells in high-fat-diet mice. Obesity (Silver Spring) 25, 581-590, doi:10.1002/oby.21769 (2017).

19 Klotz, L. et al. The nuclear receptor PPAR gamma selectively inhibits Th17 differentiation in a T cell-intrinsic fashion and suppresses CNS autoimmunity. $J$ Exp Med 206, 2079-2089, doi:10.1084/jem.20082771 (2009).

20 Lewis, J. D. et al. An open-label trial of the PPAR-gamma ligand rosiglitazone for active ulcerative colitis. Am J Gastroenterol 96, 3323-3328, doi:10.1111/j.15720241.2001.05333.x (2001).

21 Huang, Y. et al. Pioglitazone Attenuates Experimental Colitis-Associated Hyperalgesia through Improving the Intestinal Barrier Dysfunction. Inflammation, doi:10.1007/s10753-019-01138-3 (2020).

22 Liang, H. L. \& Ouyang, Q. A clinical trial of combined use of rosiglitazone and 5aminosalicylate for ulcerative colitis. World $J$ Gastroenterol 14, 114-119, doi:10.3748/wjg.14.114 (2008).

23 Ogawa, S., Takeuchi, K. \& Ito, S. Plasma BNP levels in the treatment of type 2 diabetes with pioglitazone. J Clin Endocrinol Metab 88, 3993-3996, doi:10.1210/jc.2002021765 (2003).

24 Liao, H. W. et al. Pioglitazone and cardiovascular outcomes in patients with insulin resistance, pre-diabetes and type 2 diabetes: a systematic review and meta-analysis. BMJ Open 7, e013927, doi:10.1136/bmjopen-2016-013927 (2017). 
Cheng, H. S. et al. Exploration and Development of PPAR Modulators in Health and Disease: An Update of Clinical Evidence. Int J Mol Sci 20, doi:10.3390/ijms20205055 (2019).

26 Decara, J. et al. Peroxisome Proliferator-Activated Receptors: Experimental Targeting for the Treatment of Inflammatory Bowel Diseases. Front Pharmacol 11, 730, doi:10.3389/fphar.2020.00730 (2020).

27 Dou, X., Xiao, J., Jin, Z. \& Zheng, P. Peroxisome proliferator-activated receptor- $\gamma$ is downregulated in ulcerative colitis and is involved in experimental colitis-associated neoplasia. Oncol Lett 10, 1259-1266, doi:10.3892/ol.2015.3397 (2015).

28 Dubuquoy, L. et al. Impaired expression of peroxisome proliferator-activated receptor gamma in ulcerative colitis. Gastroenterology 124, 1265-1276, doi:10.1016/s00165085(03)00271-3 (2003).

29 Poliska, S. et al. Association of peroxisome proliferator-activated receptor gamma polymorphisms with inflammatory bowel disease in a Hungarian cohort. Inflamm Bowel Dis 18, 472-479, doi:10.1002/ibd.21798 (2012).

30 Hugot, J. P. PPAR and Crohn's disease: another piece of the puzzle? Gastroenterology 128, 500-503, doi:10.1053/j.gastro.2004.12.041 (2005).

31 Zhang, Z. F., Yang, N., Zhao, G., Zhu, L. \& Wang, L. X. Association between the Pro12Ala polymorphism of peroxisome proliferator-activated receptor gamma 2 and inflammatory bowel disease: a meta-analysis. PLoS One 7, e30551, doi:10.1371/journal.pone.0030551 (2012).

32 Takada, I. \& Makishima, M. Peroxisome proliferator-activated receptor agonists and antagonists: a patent review (2014-present). Expert Opin Ther Pat 30, 1-13, doi:10.1080/13543776.2020.1703952 (2020).

33 Mirza, A. Z., Althagafi, II \& Shamshad, H. Role of PPAR receptor in different diseases and their ligands: Physiological importance and clinical implications. Eur J Med Chem 166, 502-513, doi:10.1016/j.ejmech.2019.01.067 (2019).

34 Jain, M. R. et al. Saroglitazar, a novel PPAR $\alpha / \gamma$ agonist with predominant PPAR $\alpha$ activity, shows lipid-lowering and insulin-sensitizing effects in preclinical models. Pharmacol Res Perspect 3, e00136, doi:10.1002/prp2.136 (2015).

35 Kim, M. K. et al. PAR-5359, a well-balanced PPARalpha/gamma dual agonist, exhibits equivalent antidiabetic and hypolipidemic activities in vitro and in vivo. Eur $J$ Pharmacol 595, 119-125, doi:10.1016/j.ejphar.2008.07.066 (2008).

36 Kim, D. K. et al. Liquid chromatography-tandem mass spectrometry of a new PPARalpha/gamma dual agonist PAR-5359 in rat plasma. Arch Pharm Res 32, 17431748, doi:10.1007/s12272-009-2212-z (2009).

37 Brown, P. J. et al. Identification of a subtype selective human PPARalpha agonist through parallel-array synthesis. Bioorg Med Chem Lett 11, 1225-1227, doi:10.1016/s0960-894x(01)00188-3 (2001).

38 Information, N. C. f. B. PubChem Compound Summary for CID 4829, Pioglitazone, 2020).

39 Koroleva, E. P. et al. Citrobacter rodentium-induced colitis: A robust model to study mucosal immune responses in the gut. $J$ Immunol Methods 421, 61-72, doi:10.1016/j.jim.2015.02.003 (2015).

40 Bosman, E. S., Chan, J. M., Bhullar, K. \& Vallance, B. A. Investigation of Host and Pathogen Contributions to Infectious Colitis Using the Citrobacter rodentium Mouse Model of Infection. Methods Mol Biol 1422, 225-241, doi:10.1007/978-1-4939-3603821 (2016).

41 Bhinder, G. et al. The Citrobacter rodentium mouse model: studying pathogen and host contributions to infectious colitis. J Vis Exp, e50222, doi:10.3791/50222 (2013). 
42 Krause, P. et al. IL-10-producing intestinal macrophages prevent excessive antibacterial innate immunity by limiting IL-23 synthesis. Nat Commun 6, 7055, doi:10.1038/ncomms8055 (2015).

43 Jiminez, J. A., Uwiera, T. C., Douglas Inglis, G. \& Uwiera, R. R. Animal models to study acute and chronic intestinal inflammation in mammals. Gut Pathog 7, 29, doi:10.1186/s13099-015-0076-y (2015).

44 Kozaiwa, K. et al. Identification of a quantitative trait locus for ileitis in a spontaneous mouse model of Crohn's disease: SAMP1/YitFc. Gastroenterology 125, 477-490, doi:10.1016/s0016-5085(03)00876-X (2003).

45 Qi, Y. et al. PPARalpha-dependent exacerbation of experimental colitis by the hypolipidemic drug fenofibrate. Am J Physiol Gastrointest Liver Physiol 307, G564573, doi:10.1152/ajpgi.00153.2014 (2014).

$46 \mathrm{Gu}$, X. et al. GC-MS metabolomics on PPARalpha-dependent exacerbation of colitis. Mol Biosyst 11, 1329-1337, doi:10.1039/c5mb00048c (2015).

47 Mohapatra, S. K. et al. Immunoregulatory actions of epithelial cell PPAR gamma at the colonic mucosa of mice with experimental inflammatory bowel disease. PLoS One 5, e10215, doi:10.1371/journal.pone.0010215 (2010).

48 Hontecillas, R. et al. Immunoregulatory mechanisms of macrophage PPAR- $\gamma$ in mice with experimental inflammatory bowel disease. Mucosal Immunol 4, 304-313, doi:10.1038/mi.2010.75 (2011).

49 Guri, A. J., Mohapatra, S. K., Horne, W. T., 2nd, Hontecillas, R. \& Bassaganya-Riera, $\mathrm{J}$. The role of $\mathrm{T}$ cell PPAR gamma in mice with experimental inflammatory bowel disease. BMC Gastroenterol 10, 60, doi:10.1186/1471-230x-10-60 (2010).

50 Boudeau, J., Glasser, A. L., Masseret, E., Joly, B. \& Darfeuille-Michaud, A. Invasive ability of an Escherichia coli strain isolated from the ileal mucosa of a patient with Crohn's disease. Infect Immun 67, 4499-4509 (1999).

51 Pavlou, S., Wang, L., Xu, H. \& Chen, M. Higher phagocytic activity of thioglycollateelicited peritoneal macrophages is related to metabolic status of the cells. J Inflamm (Lond) 14, 4, doi:10.1186/s12950-017-0151-x (2017).

52 Layoun, A., Samba, M. \& Santos, M. M. Isolation of murine peritoneal macrophages to carry out gene expression analysis upon Toll-like receptors stimulation. J Vis Exp, e52749, doi:10.3791/52749 (2015).

53 Tan, H. Y. et al. The Reactive Oxygen Species in Macrophage Polarization: Reflecting Its Dual Role in Progression and Treatment of Human Diseases. Oxid Med Cell Longev 2016, 2795090, doi:10.1155/2016/2795090 (2016).

54 Tan, H.-Y. et al. The Reactive Oxygen Species in Macrophage Polarization: Reflecting Its Dual Role in Progression and Treatment of Human Diseases. Oxidative Medicine and Cellular Longevity 2016, 2795090, doi:10.1155/2016/2795090 (2016).

55 Segal, A. W. Studies on patients establish Crohn's disease as a manifestation of impaired innate immunity. J Intern Med 286, 373-388, doi:10.1111/joim.12945 (2019).

56 Baillie, J. K. et al. Analysis of the human monocyte-derived macrophage transcriptome and response to lipopolysaccharide provides new insights into genetic aetiology of inflammatory bowel disease. PLoS Genet 13, e1006641, doi:10.1371/journal.pgen.1006641 (2017).

57 Quezada, S. M., McLean, L. P. \& Cross, R. K. Adverse events in IBD therapy: the 2018 update. Expert Rev Gastroenterol Hepatol 12, 1183-1191, doi:10.1080/17474124.2018.1545574 (2018).

58 Harris, M. S., Wichary, J., Zadnik, M. \& Reinisch, W. Competition for Clinical Trials in Inflammatory Bowel Diseases. Gastroenterology 157, 1457-1461.e1452, doi:10.1053/j.gastro.2019.08.020 (2019). 
59 Zhou, X. et al. PPARalpha-UGT axis activation represses intestinal FXR-FGF15 feedback signalling and exacerbates experimental colitis. Nat Commun 5, 4573, doi:10.1038/ncomms5573 (2014).

60 Manoharan, I. et al. Homeostatic PPARalpha Signaling Limits Inflammatory Responses to Commensal Microbiota in the Intestine. J Immunol 196, 4739-4749, doi:10.4049/jimmunol.1501489 (2016).

61 Azuma, Y. T. et al. PPARalpha contributes to colonic protection in mice with DSSinduced colitis. Int Immunopharmacol 10, 1261-1267, doi:10.1016/j.intimp.2010.07.007 (2010).

62 Lewis, J. D. et al. Rosiglitazone for active ulcerative colitis: a randomized placebocontrolled trial. Gastroenterology 134, 688-695, doi:10.1053/j.gastro.2007.12.012 (2008).

63 Pedersen, G. \& Brynskov, J. Topical rosiglitazone treatment improves ulcerative colitis by restoring peroxisome proliferator-activated receptor-gamma activity. Am J Gastroenterol 105, 1595-1603, doi:10.1038/ajg.2009.749 (2010).

64 Chawla, A. Control of macrophage activation and function by PPARs. Circ Res 106, 1559-1569, doi:10.1161/circresaha.110.216523 (2010).

65 Leopold Wager, C. M., Arnett, E. \& Schlesinger, L. S. Macrophage nuclear receptors: Emerging key players in infectious diseases. PLoS Pathog 15, e1007585, doi:10.1371/journal.ppat.1007585 (2019).

66 Singh, S., Loke, Y. K. \& Furberg, C. D. Long-term use of thiazolidinediones and the associated risk of pneumonia or lower respiratory tract infection: systematic review and meta-analysis. Thorax 66, 383-388, doi:10.1136/thx.2010.152777 (2011).

67 Teissier, E. et al. Peroxisome proliferator-activated receptor alpha induces NADPH oxidase activity in macrophages, leading to the generation of LDL with PPAR-alpha activation properties. Circ Res 95, 1174-1182, doi:10.1161/01.Res.0000150594.95988.45 (2004).

68 Guri, A. J., Evans, N. P., Hontecillas, R. \& Bassaganya-Riera, J. T cell PPARgamma is required for the anti-inflammatory efficacy of abscisic acid against experimental IBD. J Nutr Biochem 22, 812-819, doi:10.1016/j.jnutbio.2010.06.011 (2011).

69 Hontecillas, R. \& Bassaganya-Riera, J. Expression of PPAR gamma in intestinal epithelial cells is dispensable for the prevention of colitis by dietary abscisic acid. Espen j 7, e189-e195, doi:10.1016/j.clnme.2012.07.002 (2012). 


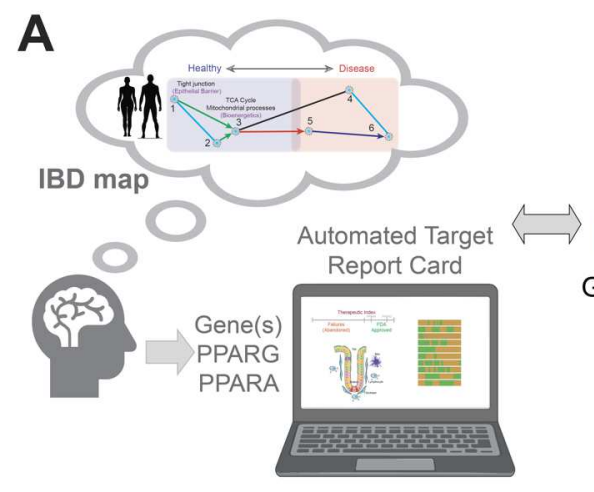

C GSE73661 GSE16879

GSE59071

GSE48958

D

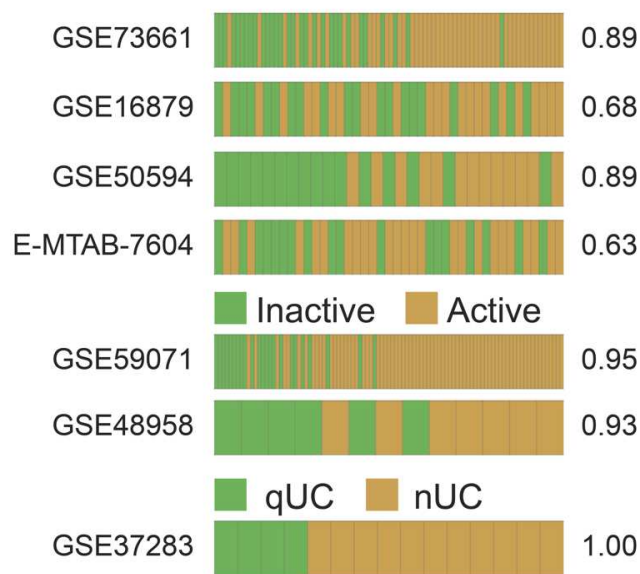<smiles>[10BH]</smiles>

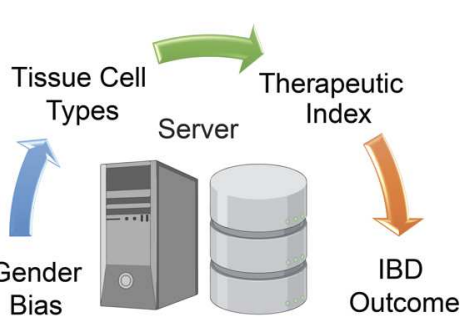

Database

Mouse

Model

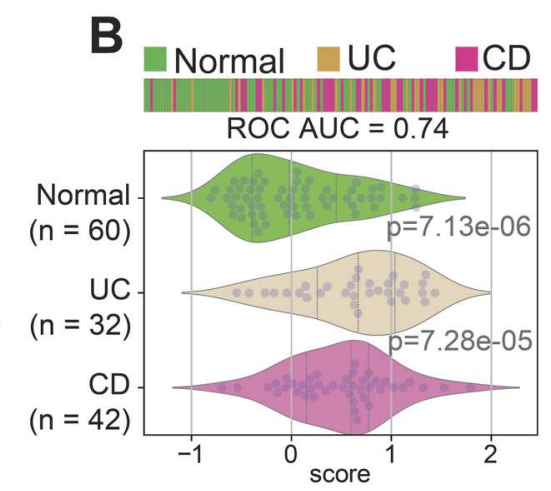

E

Mouse Model Dataset Normal $\square$ IBD $\begin{aligned} & \text { ROC } \\ & \text { AUC }\end{aligned}$ DSS (bulk) GSE42768

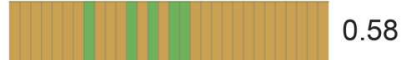

DSS (epithelium) E-MTAB-5249

TNBS

GSE53835

Citrobacter

ACT

ACT

IL10 -/- (epithelium) GSE39859

IL10 -/- (bulk)

GSE39859

TNFR1 -/-

GSE107933

TNFR2 -/-

GSE65408
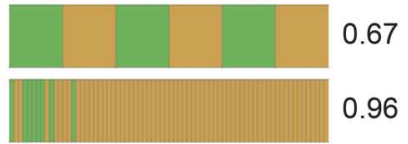

GSE90577

GSE87317
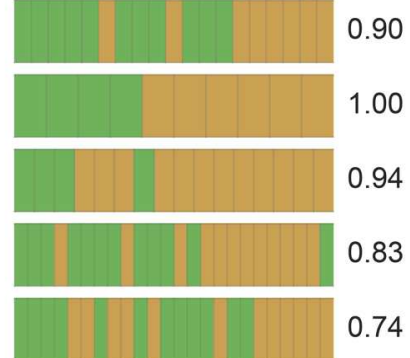

0.74

0.56

0.56

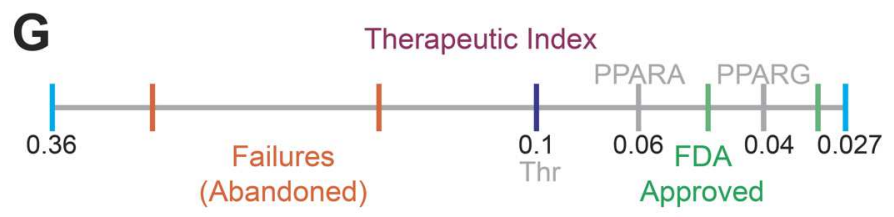

$\mathbf{F}$

Databases

GSE13067,GSE14333,

GSE17538,GSE31595,

GSE37892,GSE33113

GSE134312 Mac

GSE63626 Fibroblast

Boolean Implication

Analysis

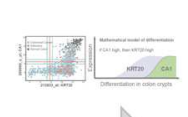

Predict Cell Type Specific Expression

GSE24759 @olymphocyte

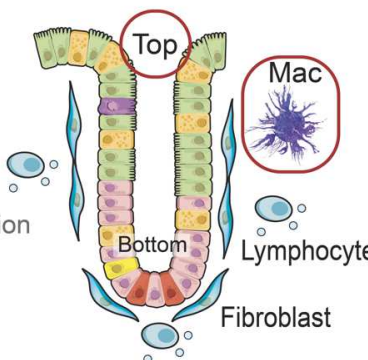

$\mathrm{H}$

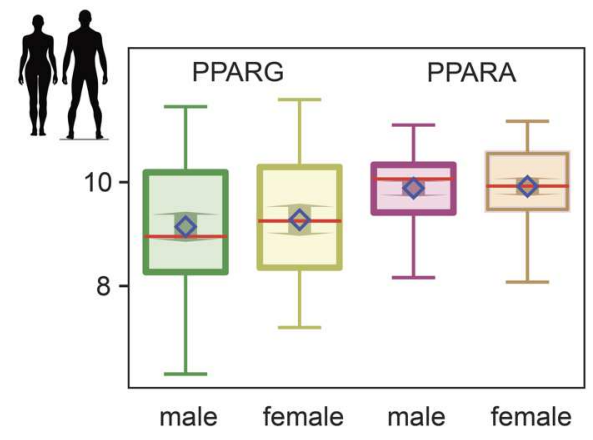

Figure 1: Network-rationalized target identification and study design. (A) Schematic displays the overall computationally guided study design. An interactive web-based platform allows the querying of paths of gene clusters in the IBD map [ ${ }^{9}$; see Supplementary Fig. 1] to pick high-value targets with a few mouse clicks and 
generate a comprehensive automated target 'report card'. The components of a 'target report card' is shown (right): predicted 'therapeutic index' (likelihood of Phase III success), IBD outcome (prognostic potential in UC and/or CD), network-prioritized mouse model, estimation of gender bias and predicted tissue cell type of action. (B-H) Components of a target report card for PPARA and PPARG are displayed. Bar plot (B; top) displays the rank ordering of normal vs ulcerative colitis (UC) /Crohn's Disease (CD) patient samples using the average gene expression patterns of the two genes: PPARG/PPARA. ROC-AUC statistics were measured for determining the classification strength of normal vs IBD. Bar plots (B; top) and violin plots (B; bottom) display the differences in the average expression of the two genes in normal, UC and CD samples in the test cohort used to build the IBDmap. Bar plots in panel C-D show the rank ordering of either normal vs IBD samples (C) or responder vs nonresponder (R $v s$. NR; D), or active vs inactive disease, or neoplastic progression in quiescent UC (qUC vs. nUC; D) across numerous cohorts based on gene expression patterns of PPARG and PPARA, from high to low, left to right. Classification strength within each cohort is measured using ROC-AUC analyses. Bar plots in panel E show the rank ordering of either normal vs IBD samples across numerous published murine models of IBD based on gene expression patterns of PPARG and PPARA as in D. ACT = adoptive T cell transfer. Classification strength within each cohort is measured using ROC-AUC analyses. Bulk = whole distal colon; epithelium $=$ sorted epithelial cells. Schematic in F summarizes the computational prediction of the cell type of action for potential PPARA/G targeted therapy, as determined using Boolean implication analysis. GSEID\# of multiple publicly available databases of the different cell types and colorectal datasets used to make sure predictions are cited. Red boxes/circles denote that PPARA/G-targeted therapeutics are predicted to work on monocytes/macrophages and crypt-top enterocytes. Computationally generated therapeutic index (see Methods) is represented as a line graph in G. The annotated numbers represent Boolean implication statistics. PPARA and PPARG align with FDA approved targets on the right of threshold (0.1). Two FDA approved targets (green; ITGB1, 0.046; JAK2, 0.032), two abandoned targets (red; SMAD7, 0.33; IL11, 0.16), PPARA (grey, 0.064), PPARG (grey, 0.04), and the threshold (black, 0.1) are shown in the scale. Box plot in panel H shows that the level of PPARA/G expression is similar in the colons of both genders in health and in IBD, and hence, PPAR $\alpha / \gamma$-targeted therapeutics are predicted to have little/no gender predilection. 

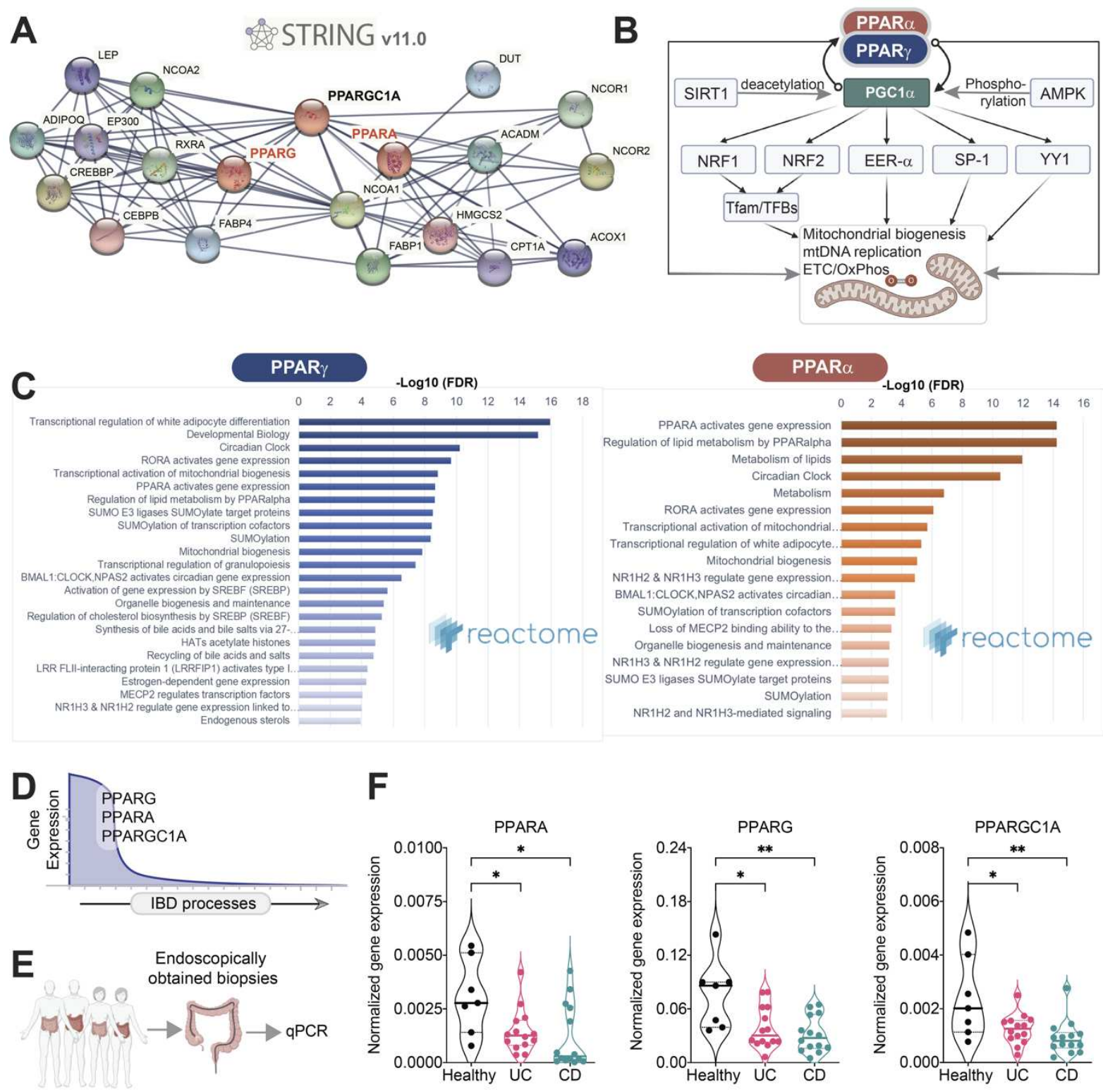

(A) A protein-protein interaction network (i.e., interactomes) for PPAR $\alpha$ and PPAR $\gamma$, generated using STRING v.11 (https://string-db.org). (B) Schematic summarizing the roles of PPAR $\alpha$, PPAR $\gamma$ and PGC1 $\alpha$ on mitochondria biogenesis and function (based on). PGC1- $\alpha$ emerges as a critical hub for forward feedback loops. (C) Reactome pathway analyses (www.reactome.org) on PPAR- $\alpha$ and PPAR- $\gamma$ interactomes in A show convergence on metabolism, mitochondria bioenergetics and the circadian clock. (D) Graphical visualization of the predicted changes in the expression of PPARA (PPAR- $\alpha$ ), PPARG (PPAR- $\gamma$ ) and PPARGC1A (PGC1- $\alpha$ ) genes during the progression of IBD processes (indicated with an arrow). (E) Schematic showing validation workflow; the expression of PPARA, PPARG and PPARGC1A transcript levels were assessed in the ileum/colon biopsies of IBD patients ( $\mathrm{UC}=14$ and $\mathrm{CD}=14)$ ) or healthy controls $(\mathrm{n}=7)$. (F) Violin plots display the qPCR results in $\mathrm{E}$. Results are displayed as mean \pm SEM. Significance was tested using one-way ANOVA followed by Tukey's test for multiple comparisons. Significance: ${ }^{*}, \mathrm{p}<0.05 ;{ }^{* *}, \mathrm{p}<0.01$. 
A

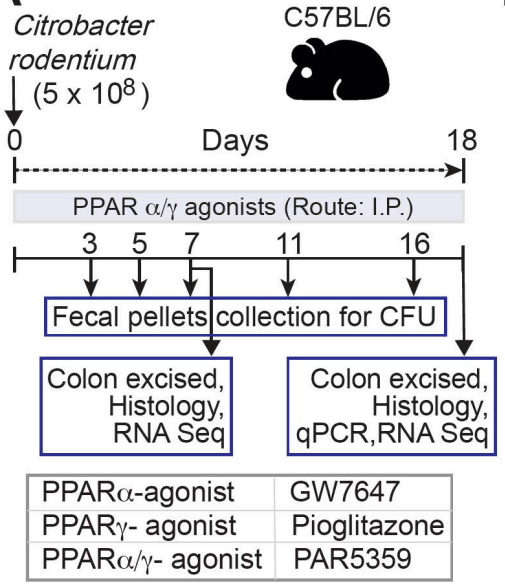

B

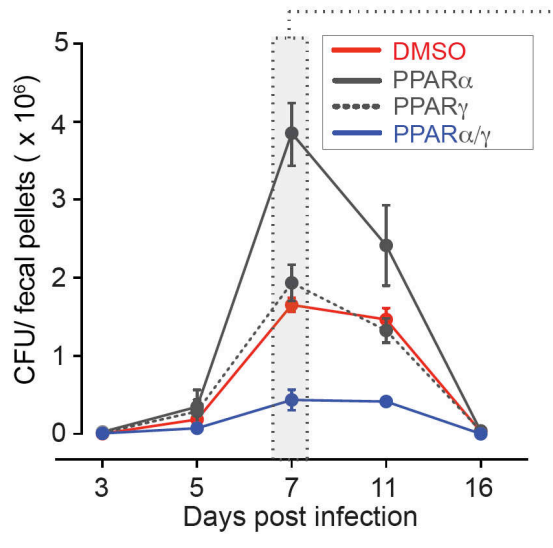

C $\stackrel{\vdots}{\vdots}$ (day \#7)

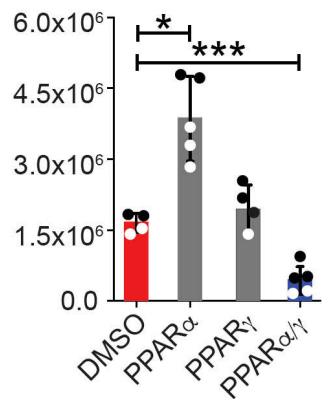

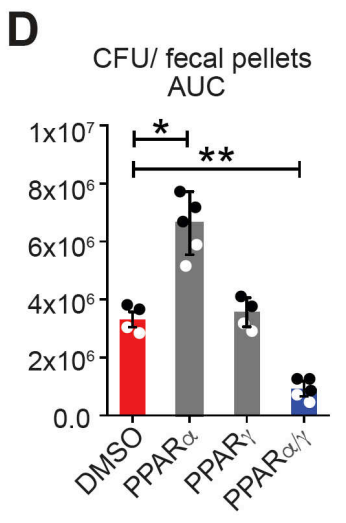

F

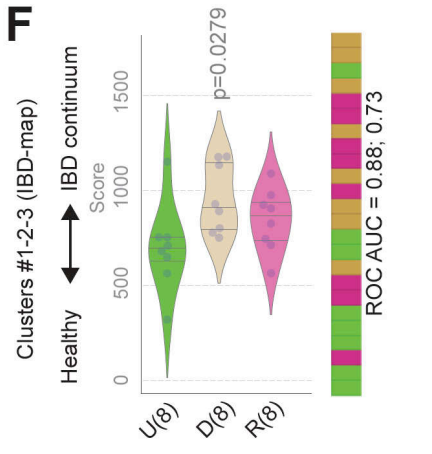

E DMSO

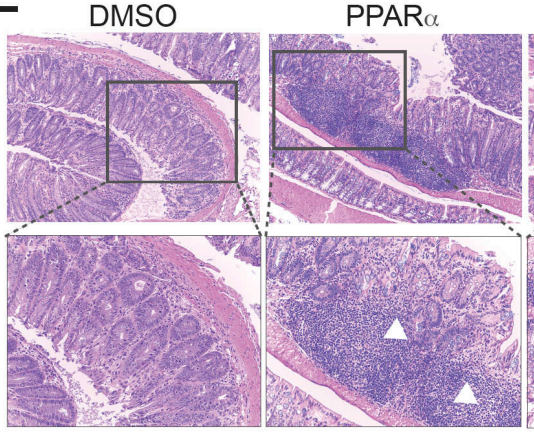
$\mathrm{PPAR}_{\gamma}$

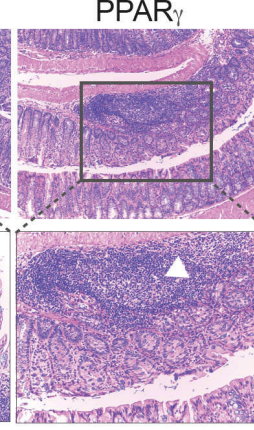

PPAR $\alpha / \gamma$

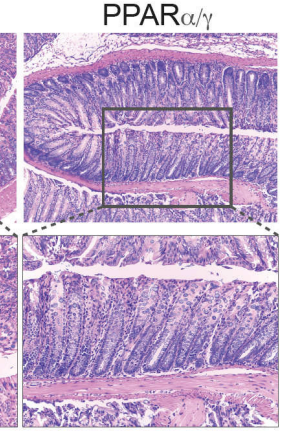

G

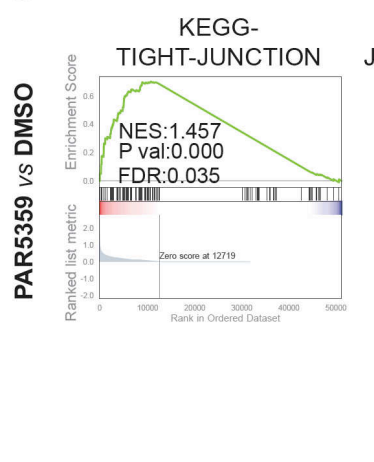

GO-ADHERENS- COATES-MACROPHAGE-
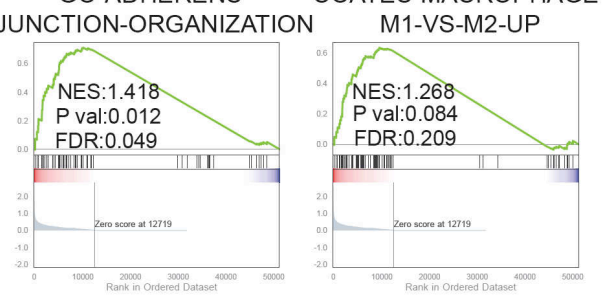

Uninfected DMSO $\square$ PAR-5359

Figure 3: PPAR $\alpha / \boldsymbol{\gamma}$ dual agonists ameliorate Citrobacter rodentium-induced infectious colitis in mice.

(A) Schematic summarizing the workflow for testing PPAR-targeted therapeutics in C. rodentium-induced colitis. Mice were gavaged with $C$. rodentium on day 0 and subsequently treated daily with PPAR agonists. Fecal pellets were collected to test viable bacterial burden, as determined by dilution plating and colony counting. Colons were excised on day 7 and 18 and analyzed using the indicated readouts. (B-D) Line graphs in B display time series of the burden of viable bacteria in feces. Scatter plots with bar graphs in C compare the peak burden of viable bacteria in feces on day 7. Scatter plots with bar graphs in D display the area under the curve (AUC) for the line graph in B. (E) Images display representative fields from H\&E-stained colon tissues. Mag = 100x (top) and 200x (bottom). White arrowheads point to immune cell infiltrates. Statistics: All results are displayed as mean \pm SEM. Significance was tested using two-way/one-way ANOVA followed by Tukey's test for multiple comparisons. Significance: $*, p<0.05 ; * *, p<0.01, * * *, p<0.001$. (F) Violin plots (left) display the deviation of expression of genes in Clusters \#1-2-3 in the IBD network, as determined by RNA Seq on murine colons. Bar plot (right) displays the rank ordering of the samples. (G) Pre-ranked GSEA based on pairwise differential expression analyses (DMSO vs PAR5359 groups) are displayed as enrichment plots for epithelial tight (left) and adherens (middle) junction signatures and balanced macrophage processes (right). See also Supplementary Fig. 7 for the Day \#7 results in the $C$. rodentium-induced colitis model, Supplementary Fig. 8 for extended GSEA analyses, and Supplementary Fig. 9 for the effect of PAR5359 on DSS-induced colitis in mice. 
A

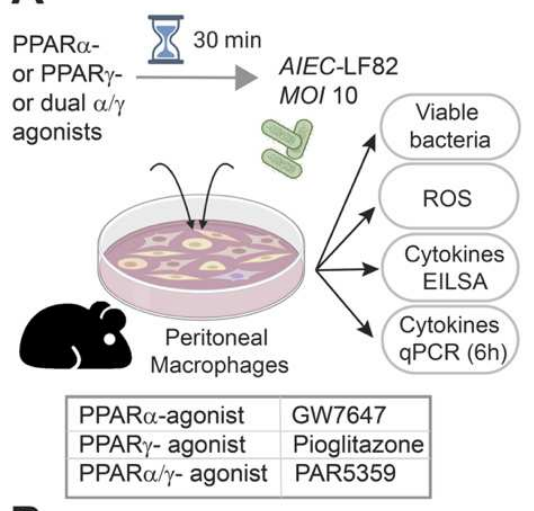

B

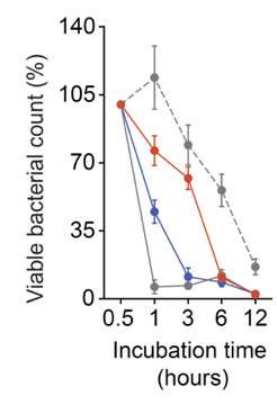

C

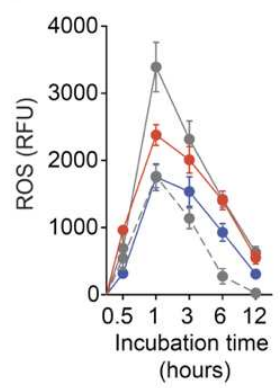

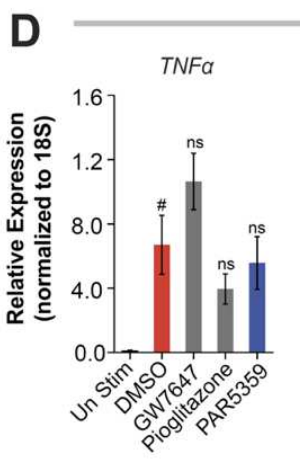
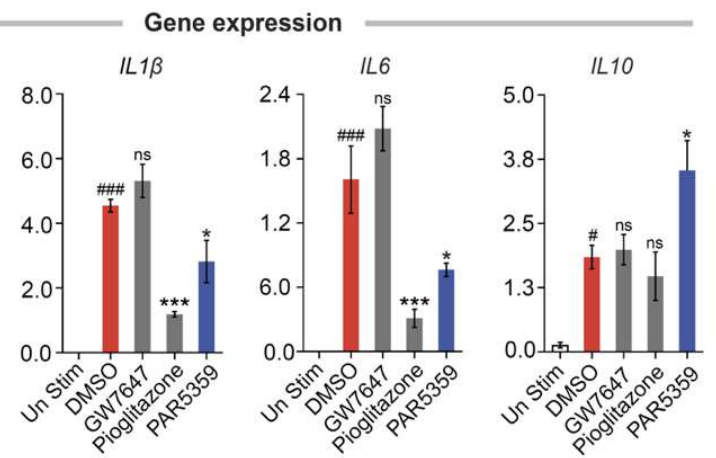

$\mathbf{E}$
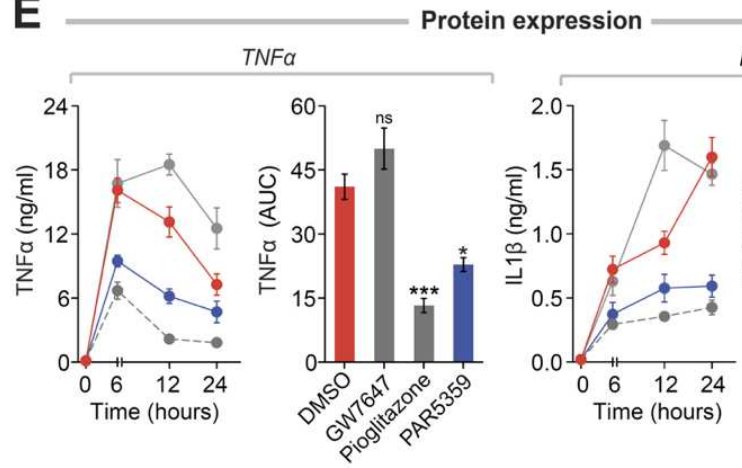

IL $1 \beta$

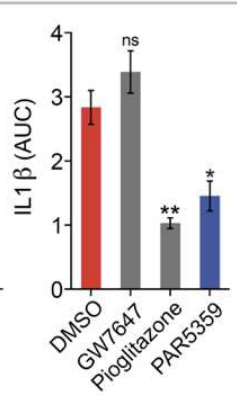

IL6
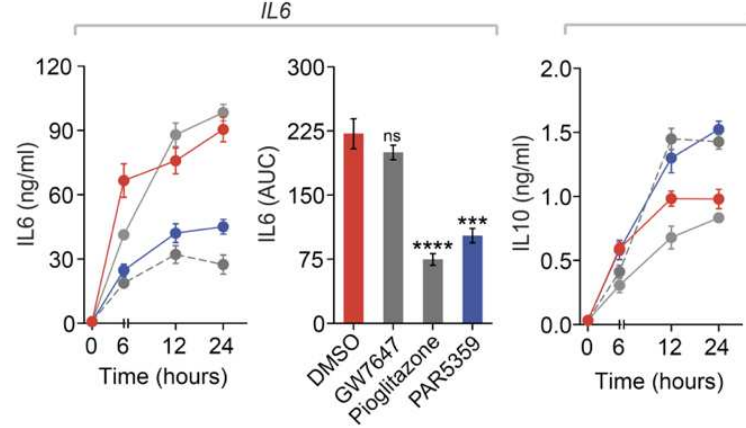

IL10
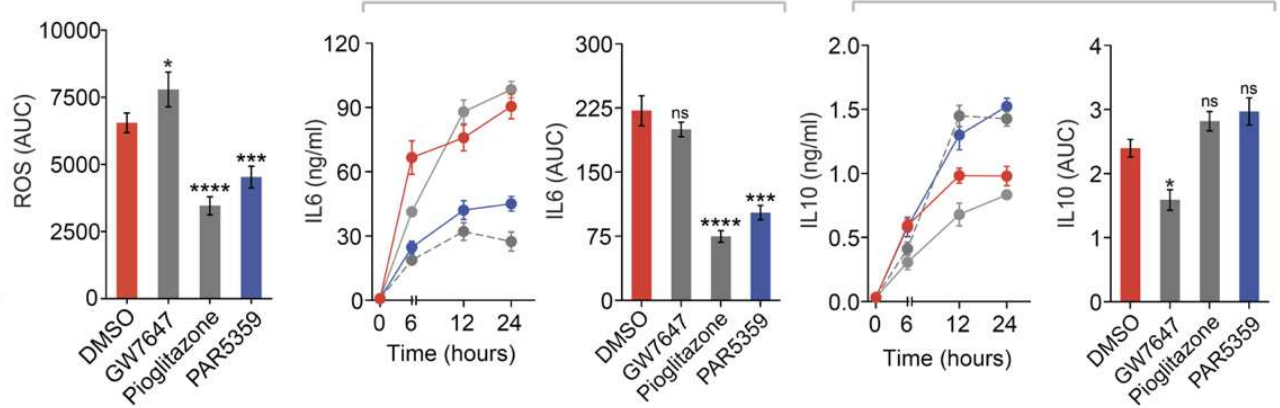

Figure 4: PPAR $\alpha$ and PPAR $\alpha / \gamma$-dual agonists enhance, whereas PPAR $\gamma$ agonist delay bacterial (AIEC-

LF82) clearance. (A) Schematic displays the experimental design and workflow. Thioglycolate-induced murine peritoneal macrophages (TG-PM) pretreated with PPAR agonists (see box, below; $20 \mathrm{nM} \mathrm{GW7647,} 10 \mu \mathrm{M}$ Pioglitazone and $1 \mu \mathrm{M}$ PAR5359) were infected with AIEC-LF82 (MOI 10) and subsequently analyzed for the bacterial count (Gentamycin protection assay), generation of cellular ROS, secretion of inflammatory cytokines (in supernatant media by ELISA) and the induction of cytokines (gene transcript analysis by qPCR). (B) Line graphs (left) display percent viable bacterial counts at indicated times after infection. Bar graphs (right) display the AUC. (C) Line graphs (left) and bar graphs (right) display the extent of ROS generation over time. (D) Bar graphs display the relative expression of transcripts of multiple cytokines (IL1 $\beta$, IL6, TNF $\alpha$ and IL10). (E) Line graphs (left) and bar graphs (right) showing the levels of secreted cytokines in the media. Statistics: All results are from at least three independent experiments and results displayed as means \pm SEM. Significance was tested using two-way/one-way ANOVA followed by Tukey's test for multiple comparisons. Significance: '\#' significance over uninfected TG-PMs and '*' shows significance over AIEC-LF82 infected cells. ns, nonsignificant, $*, p<0.05 ; * *, p<0.01, * * *, p<0.001, * * * *, p<0.0001$. See Supplementary Fig. 10 for similar bacterial clearance assays performed using Salmonella enteritica. 

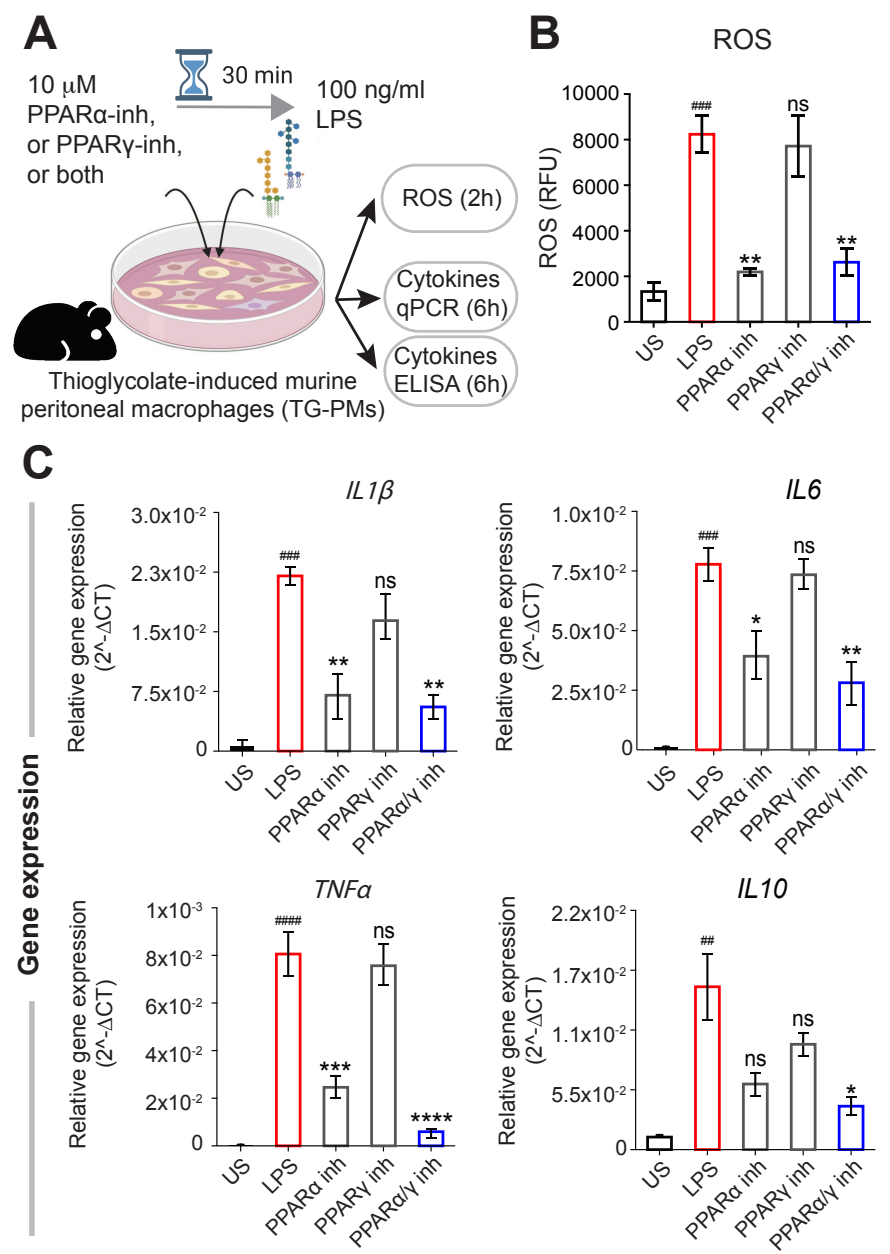

D
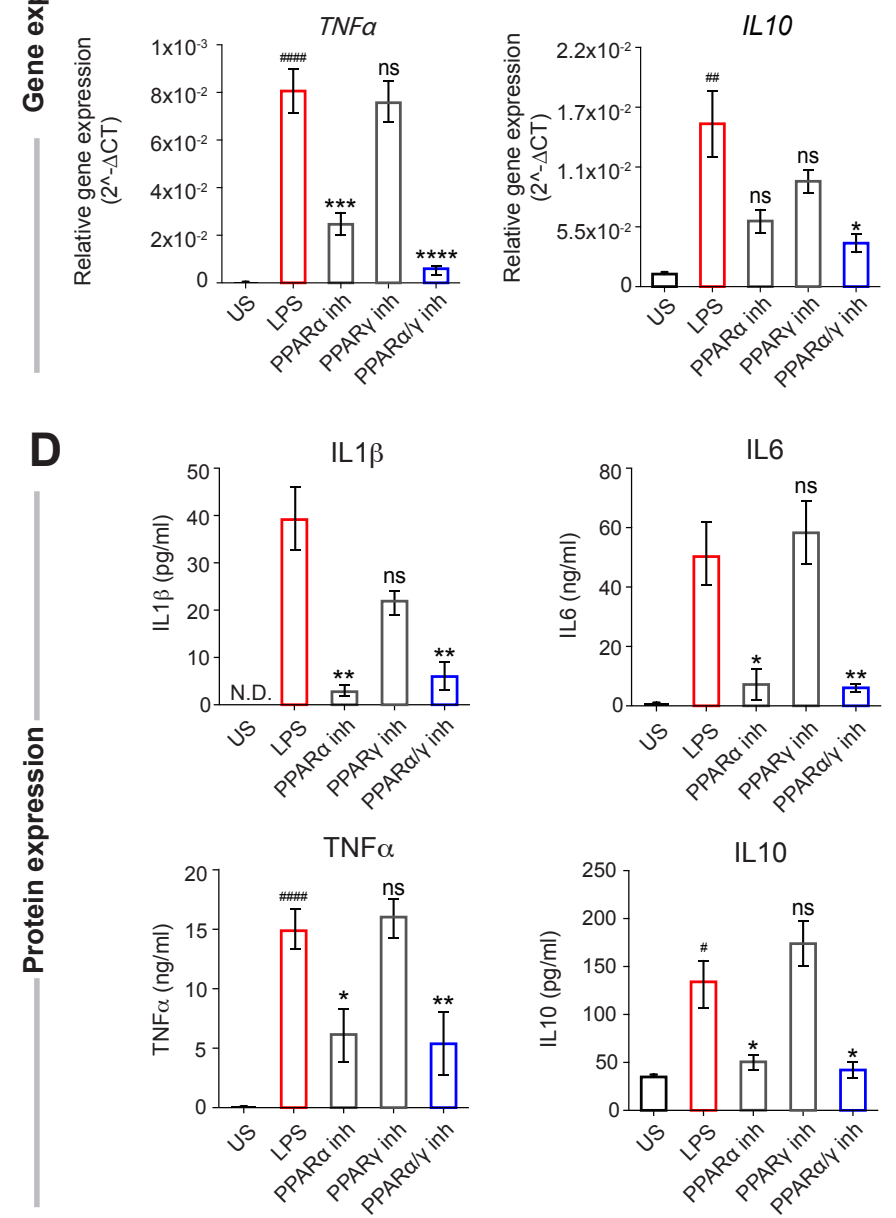

915 Figure 5: PPAR $\alpha$ but not PPAR $\gamma$ is required for induction of cellular ROS and proinflammatory cytokines.

916 (A) Schematic of experimental design. TG-PMs were pre-incubated with $10 \mu \mathrm{M}$ PPAR $\alpha$ or PPAR $\gamma$ inhibitors, either alone or in combination for $30 \mathrm{~min}$ prior to stimulation with $100 \mathrm{ng} / \mathrm{ml}$ LPS. Cells were analyzed at 2 and $6 \mathrm{~h}$ to estimate cellular ROS and cytokine induction, respectively. (B-D) Bar graphs display the levels of cellular ROS (B), relative levels of mRNA (C) and protein (D) expression of cytokines (IL1 $\beta$, IL6, TNF $\alpha$ and IL10). Statistics: Results are from three independent experiments and displayed as mean \pm SEM. One-way ANOVA followed by Tukey's test for multiple comparisons was performed to test significance. Significance: ns: nonsignificant, $*, \mathrm{p}<0.05 ; * *, \mathrm{p}<0.01, * * *, \mathrm{p}<0.001$ and $* * * *, \mathrm{p}<0.0001$ 
A

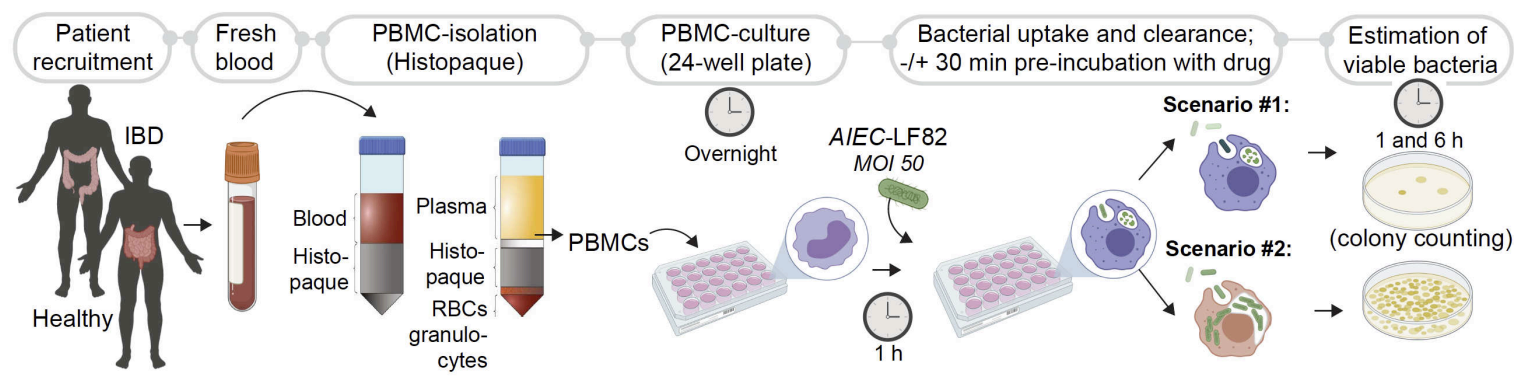

B

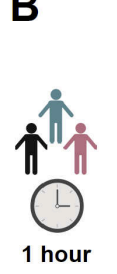

C

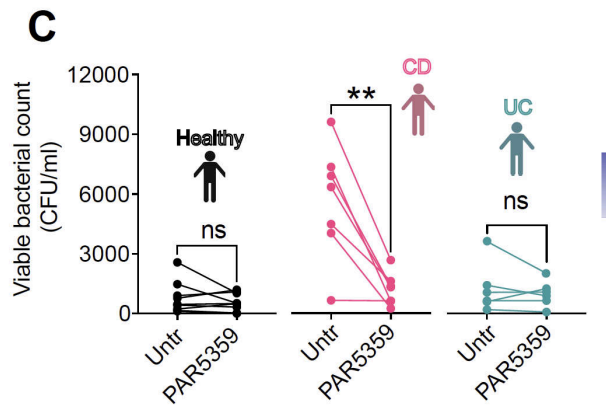

F

D

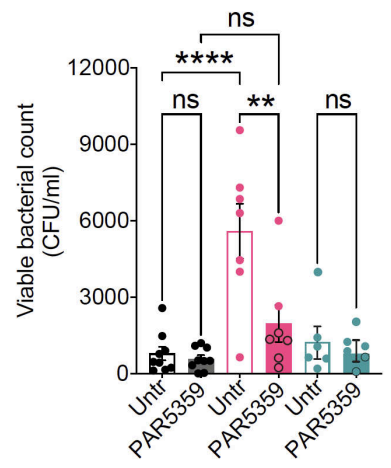

E

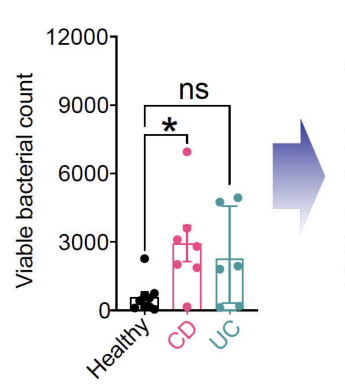

Figure 6: PPAR $\alpha / \gamma$ dual agonist, PAR5359, promotes the clearance of $A I E C$-LF82 from CD patient-derived PBMCs. (A) Schematic displays the overall experimental design using human subjects (see Table 5 for patient demographics). Peripheral blood collected from healthy, CD and UC patients was used as a source of PBMCs. PBMCs were pre-treated for $30 \mathrm{~min}$ with $1 \mu \mathrm{M}$ PPAR $\alpha / \gamma$ agonists prior to infection with AIEC-LF82 (MOI 50) for $1 \mathrm{~h}$. PBMCs were subsequently treated with gentamicin to kill extracellular microbes for $60 \mathrm{~min}(\sim \mathrm{t} 0 \mathrm{~h})$ prior to lysis and plating to determine the intracellular abundance of viable bacteria at $\mathrm{t} 1 \mathrm{~h}$ and $\mathrm{t} 6 \mathrm{~h}$, as determined by dilution plating and colony counts (see Methods for details). Bar graphs with scatter plot display the abundance of viable intracellular bacteria at $1 \mathrm{~h}(\mathbf{B})$ and $6 \mathrm{~h}(\mathbf{E})$ after infection. Paired line plots display the rate of clearance of bacteria in individual subjects at $1 \mathrm{~h}(\mathbf{C})$ and $6 \mathrm{~h}(\mathbf{F})$ after infection. Data in B-C of $1 \mathrm{~h}$ infection is combined in (D) and data from $\mathrm{E}$ and $\mathrm{F} 6 \mathrm{~h}$ infection is combined in $(\mathbf{G})$ with statistics: Results are displayed as mean $\pm \mathrm{SEM}$ (CD patient $n=7$, UC patients $=6$ and healthy $n=9$ ). Paired t-test or One-way ANOVA followed by Tukey's test for multiple comparisons was performed to test significance. Significance: ns: non-significant, $*, p<0.05 ; * *, p$ $<0.01, * * *, \mathrm{p}<0.001$ and $* * * *, \mathrm{p}<0.0001$. 


\section{Network-rationalized target identification}

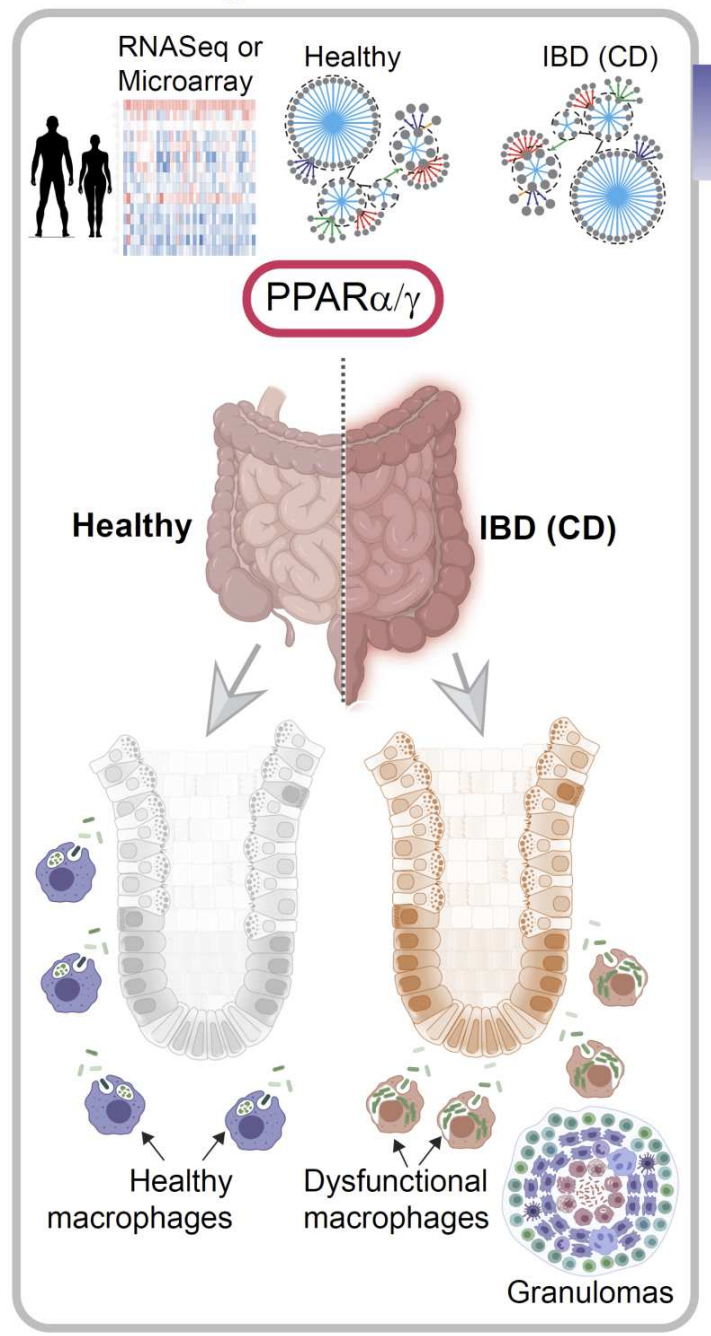

\section{Experimental determination of mechanism of action}

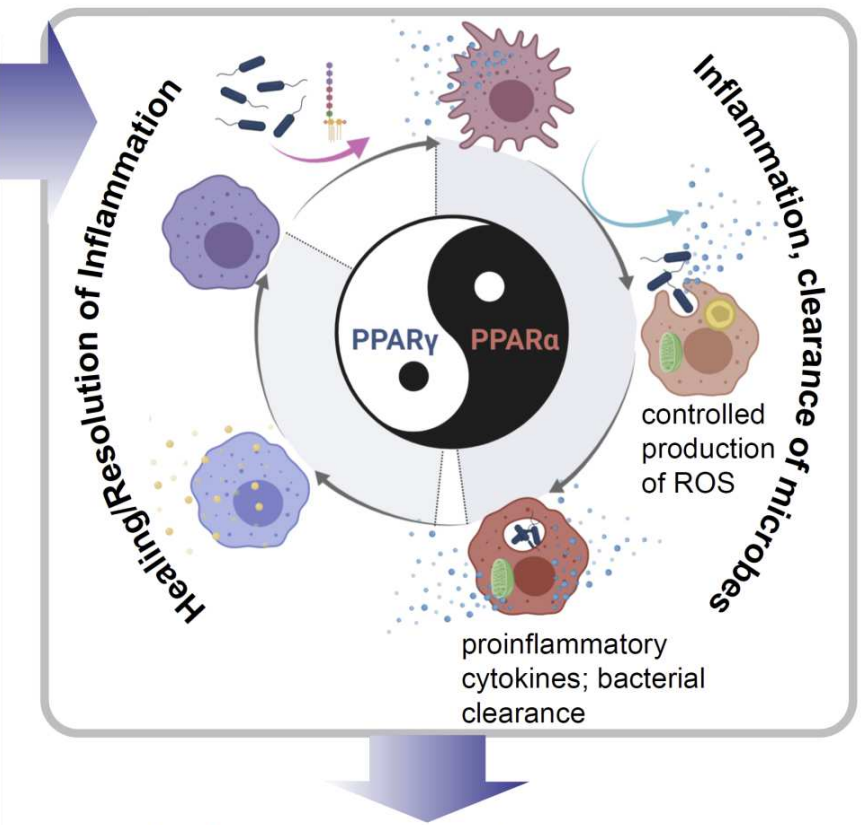

Validation in pre-clinical models

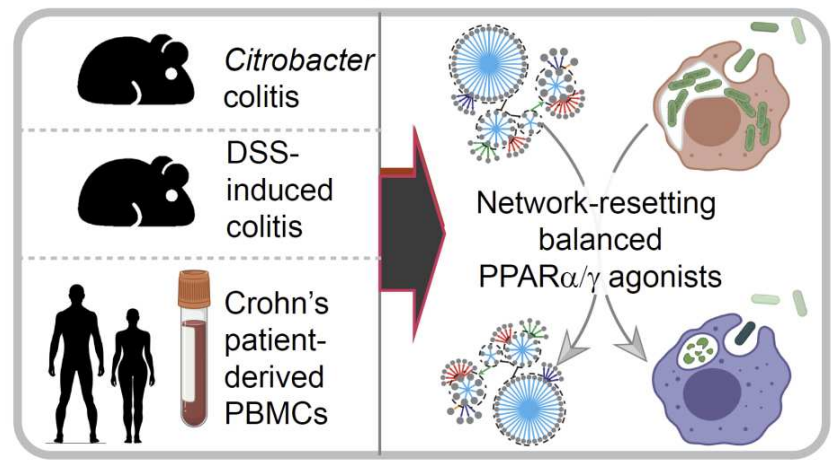

\section{Figure 7: Summary of findings and working model}

Schematic summarizes key approaches and findings of this study. First, network-rationalized target identification (Left) was performed using web-based platform that queries $>1000$ IBD datasets [ ${ }^{9}$; see Methods] that served as 'input' to create a map of gene clusters that are progressively altered in the gut in the setting of IBD. Predictions are used to guide the choice of therapeutics (dual agonists of PPAR $\alpha$ and PPAR $\gamma$ that have a balanced agonistic potential for both PPARs), the choice of animal models of IBD, predict cell types of action (macrophage processes), and finally, the subtype of IBD that could benefit most based on the cell type of action (i.e., CD). Second, experimentally determined mechanism of action studies (right, top) showed that balanced actions of both PPAR $\alpha$ and PPPAR $\gamma$ enable the induction of bacterial clearance, resolution of inflammation and healing; PPAR $\alpha$ is responsible for ROS and cytokine induction, whereas PPPAR $\gamma$ is responsible for anti-inflammatory response and healing. The dual agonistic action was superior to each agonist used alone. Third, targets validation studies (right, bottom) in murine and human models confirm the use of PPAR $\alpha / \gamma$ dual agonists for enhancing bacterial clearance and protection against colitis. When tested side-by-side in the infectious colitis model, the dual agonistic action was superior to each agonist used alone. 

A

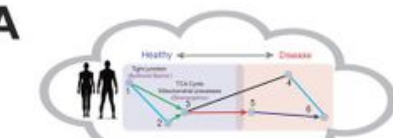

IBD map

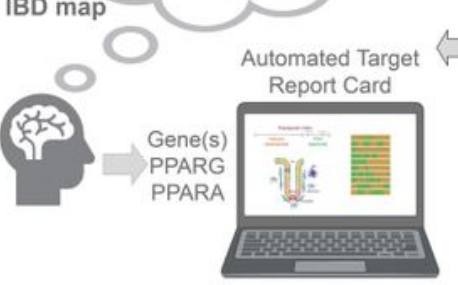

C
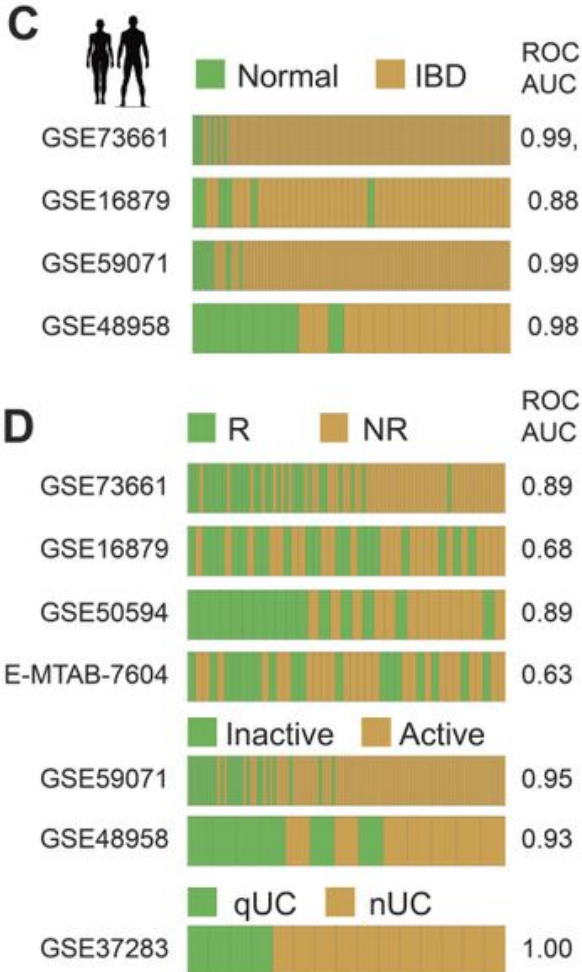

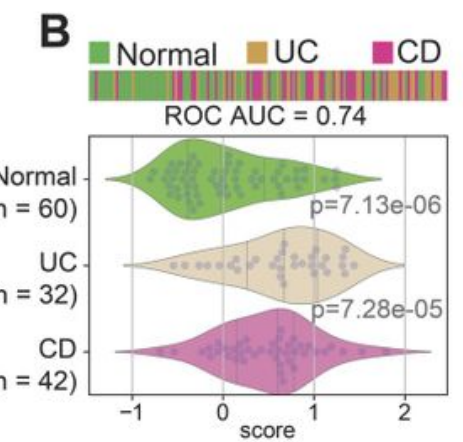

E Mouse Model I) Dataset Normal $\square$ IBD $\begin{aligned} & \text { ROC } \\ & \text { AUC }\end{aligned}$ \begin{tabular}{l|l|l} 
DSS (bulk) $\quad$ GSE42768 & 0.58
\end{tabular} DSS (epithelium) E-MTAB-5249 $\quad 0.67$ TNBS

Citrobacter

ACT

ACT

IL10 -/- (epithelium) GSE39859

IL10 -/- (bulk)

TNFR1 -/-

TNFR2 -/-
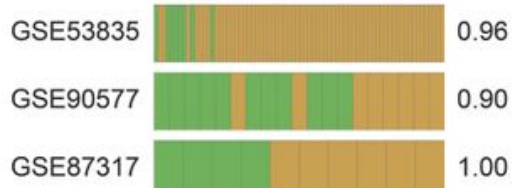

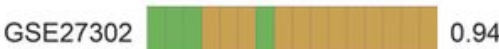

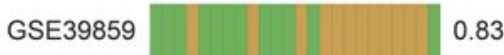

GSE39859

GSE107933

GSE65408
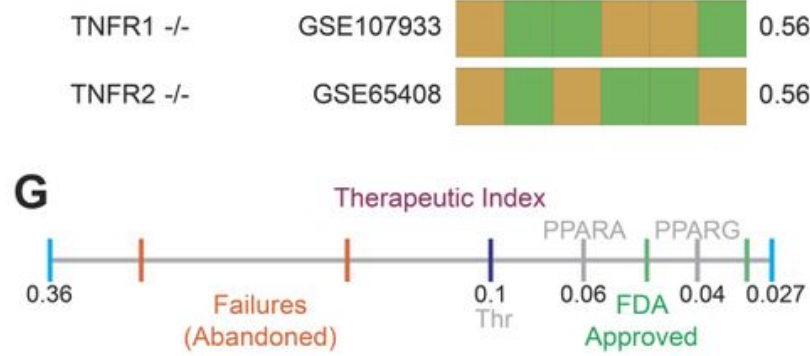

$\mathbf{F}$

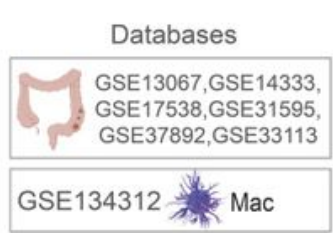

GSE63626 Fibroblast

GSE24759 ○.Lymphocyte
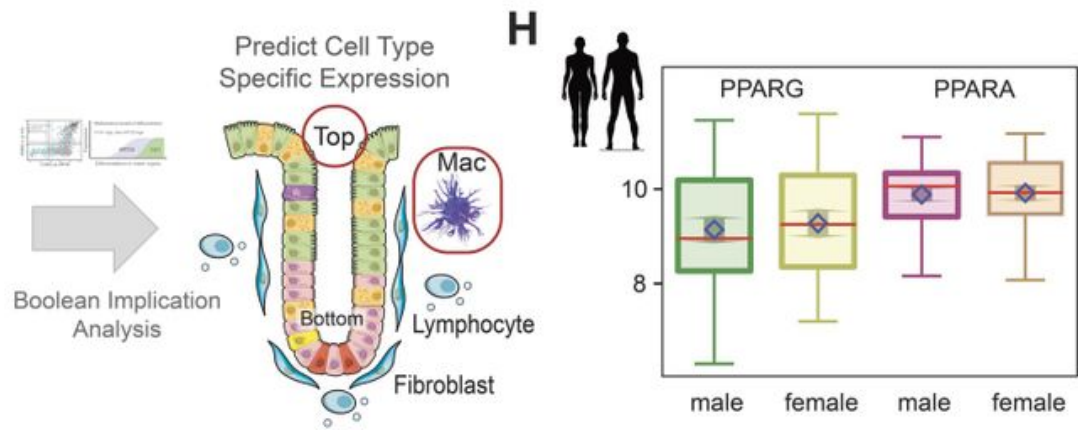

\section{Figure 1}

Network-rationalized target identification and study design. (A) Schematic displays the overall computationally guided study design. An interactive web-based platform allows the querying of paths of gene clusters in the IBD map [9; see Supplementary Fig. 1] to pick high-value targets with a few mouse 
clicks and generate a comprehensive automated target 'report card'. The components of a 'target report card' is shown (right): predicted 'therapeutic index' (likelihood of Phase III success), IBD outcome (prognostic potential in UC and/or CD), network-prioritized mouse model, estimation of gender bias and predicted tissue cell type of action. (B-H) Components of a target report card for PPARA and PPARG are displayed. Bar plot (B; top) displays the rank ordering of normal vs ulcerative colitis (UC) /Crohn's Disease (CD) patient samples using the average gene expression patterns of the two genes: PPARG/PPARA. ROCAUC statistics were measured for determining the classification strength of normal vs IBD. Bar plots (B; top) and violin plots ( $\mathrm{B}$; bottom) display the differences in the average expression of the two genes in normal, UC and CD samples in the test cohort used to build the IBD map. Bar plots in panel C-D show the rank ordering of either normal vs IBD samples (C) or responder vs non responder ( $R$ vs. NR; $D$ ), or active vs inactive disease, or neoplastic progression in quiescent UC (qUC vs. nUC; D) across numerous cohorts based on gene expression patterns of PPARG and PPARA, from high to low, left to right. Classification strength within each cohort is measured using ROC-AUC analyses. Bar plots in panel E show the rank ordering of either normal vs IBD samples across numerous published murine models of IBD based on gene expression patterns of PPARG and PPARA as in D. ACT = adoptive T cell transfer. Classification strength within each cohort is measured using ROC-AUC analyses. Bulk = whole distal colon; epithelium = sorted epithelial cells. Schematic in $\mathrm{F}$ summarizes the computational prediction of the cell type of action for potential PPARA/G targeted therapy, as determined using Boolean implication analysis. GSEID\# of multiple publicly available databases of the different cell types and colorectal datasets used to make sure predictions are cited. Red boxes/circles denote that PPARA/G-targeted therapeutics are predicted to work on monocytes/macrophages and crypt-top enterocytes. Computationally generated therapeutic index (see Methods) is represented as a line graph in $\mathrm{G}$. The annotated numbers represent Boolean implication statistics. PPARA and PPARG align with FDA approved targets on the right of threshold (0.1). Two FDA approved targets (green; ITGB1, 0.046; JAK2, 0.032), two abandoned targets (red; SMAD7, 0.33; IL11, 0.16), PPARA (grey, 0.064), PPARG (grey, 0.04), and the threshold (black, 0.1) are shown in the scale. Box plot in panel $\mathrm{H}$ shows that the level of PPARA/G expression is similar in the colons of both genders in health and in IBD, and hence, PPARa/g-targeted therapeutics are predicted to have little/no gender predilection. 

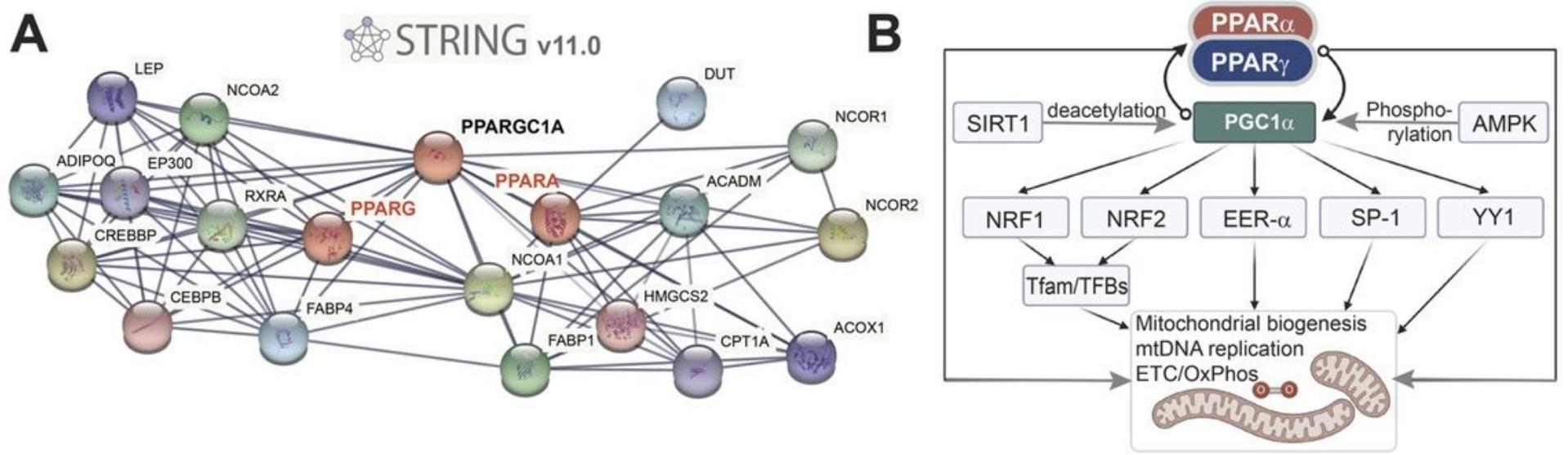

C

\section{PPAR $\gamma$}

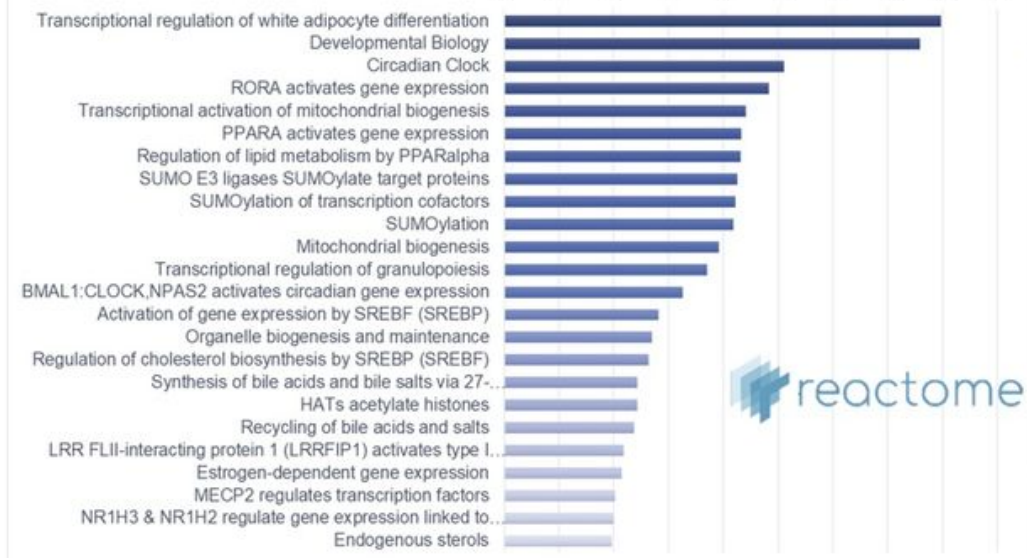

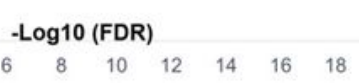

Regulation of

PPARA activates gene expression
Regulation of lipid metabolism by PPARalpha

PPAR $\alpha$
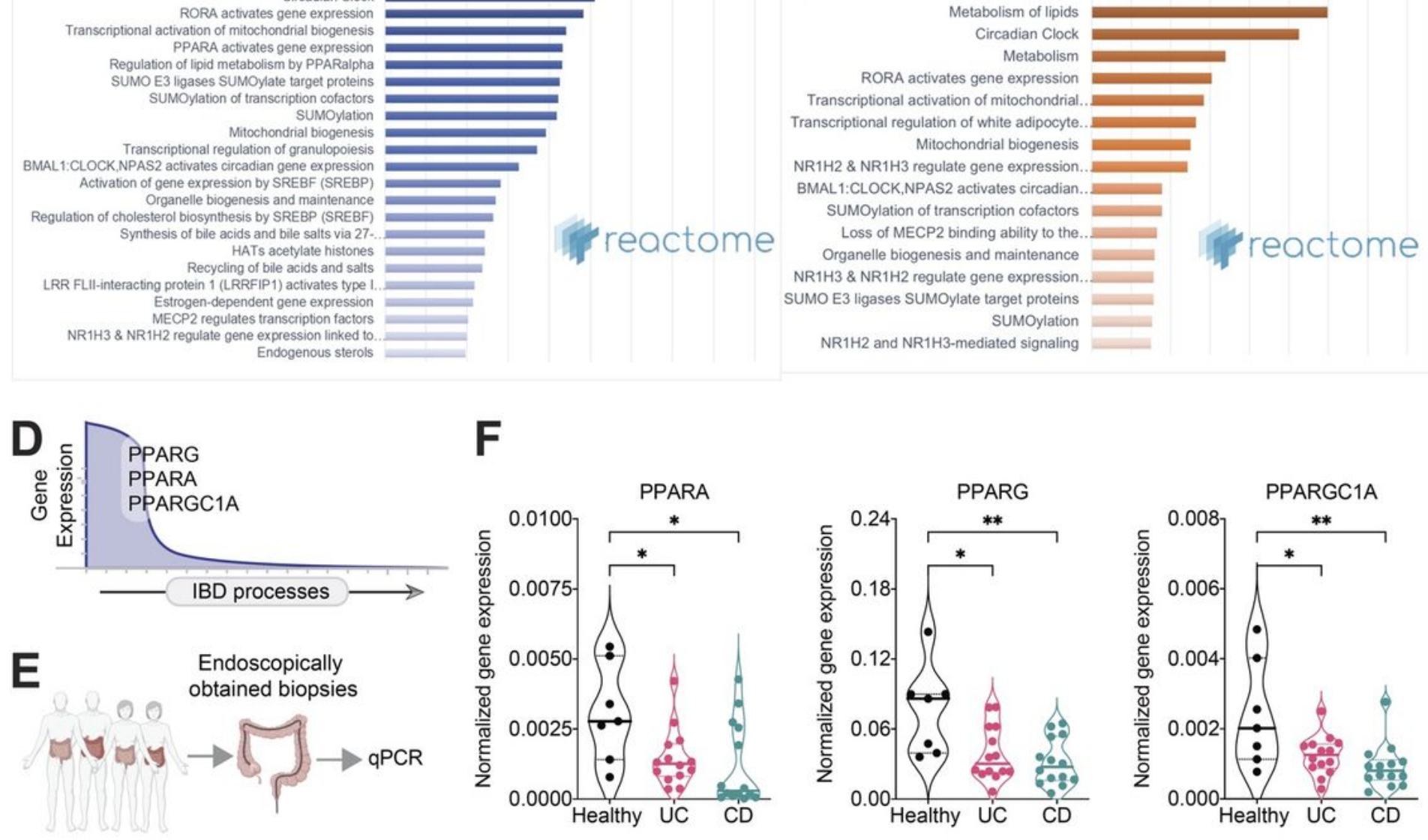

\section{Figure 2}

Rationalization of PPARa and PPARy as targets in IBD. (A) A protein-protein interaction network (i.e., interactomes) for PPARa and PPARy, generated using STRING v.11 (https://string-db.org). (B) Schematic summarizing the roles of PPARa, PPARY and PGC1a on mitochondria biogenesis and function (based on). PGC1-a emerges as a critical hub for forward feedback loops. (C) Reactome pathway analyses (www.reactome.org) on PPAR-a and PPAR-y interactomes in A show convergence on metabolism, 
mitochondria bioenergetics and the circadian clock. (D) Graphical visualization of the predicted changes in the expression of PPARA (PPAR-a), PPARG (PPAR- - ) and PPARGC1A (PGC1-a) genes during the progression of IBD processes (indicated with an arrow). (E) Schematic showing validation workflow; the expression of PPARA, PPARG and PPARGC1A transcript levels were assessed in the ileum/colon biopsies of IBD patients $(U C=14$ and $C D=14)$ ) or healthy controls $(n=7)$. ( $F)$ Violin plots display the qPCR results in E. Results are displayed as mean \pm SEM. Significance was tested using one-way ANOVA followed by Tukey's test for multiple comparisons. Significance: ${ }^{*}, p<0.05 ;{ }^{* *}, p<0.01$.

A

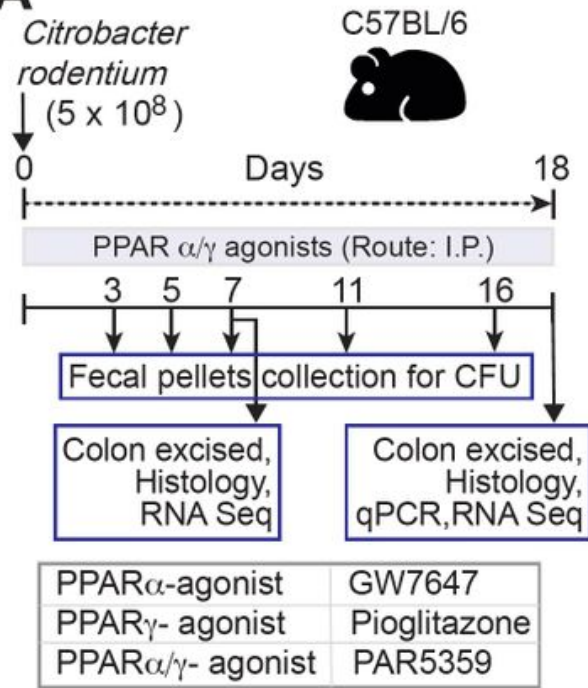

B

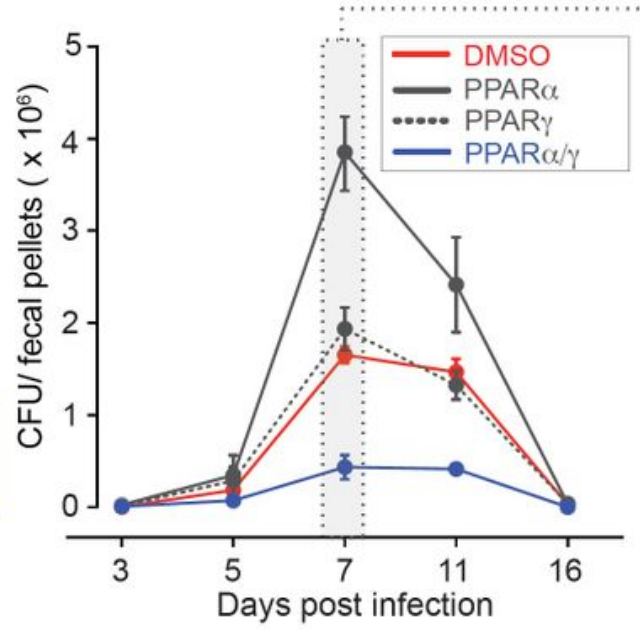

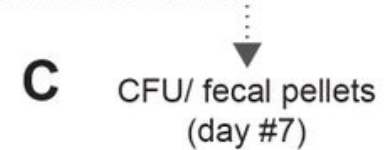

(day \#7)

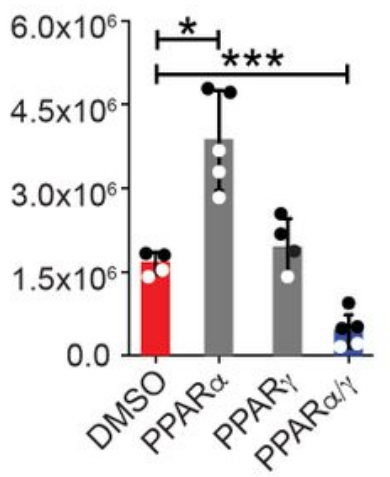

D

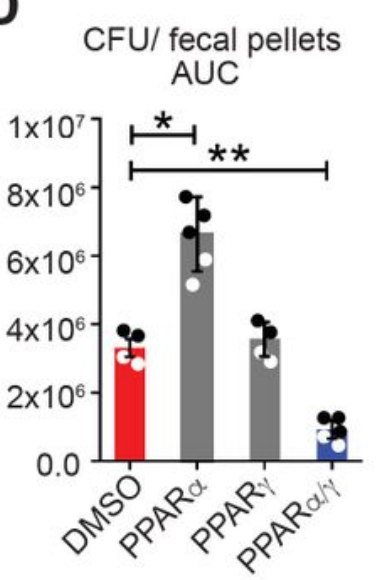

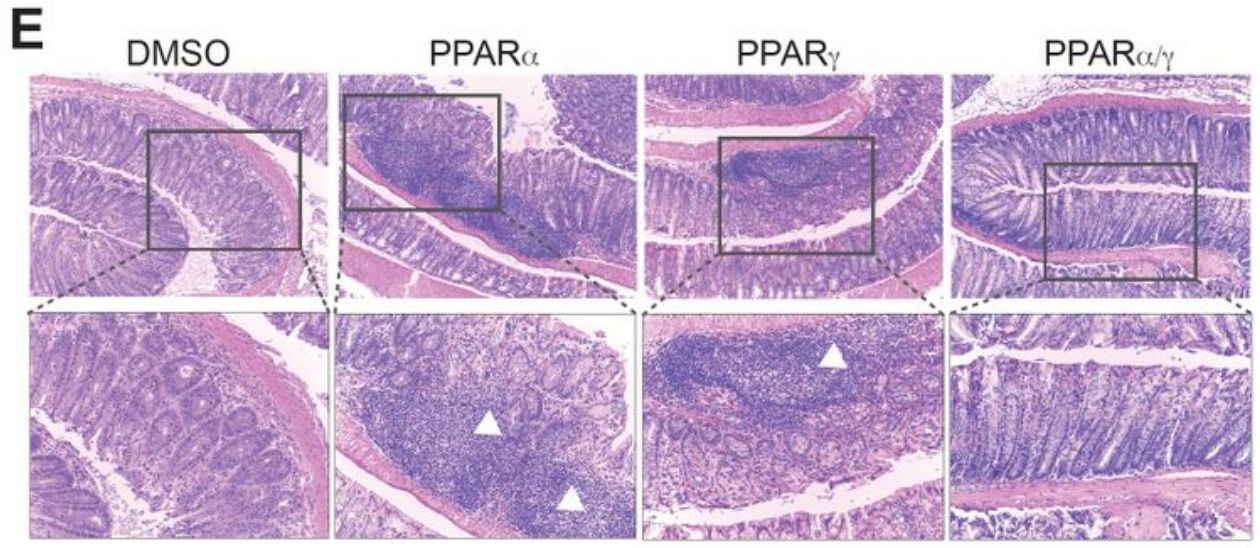

$F$

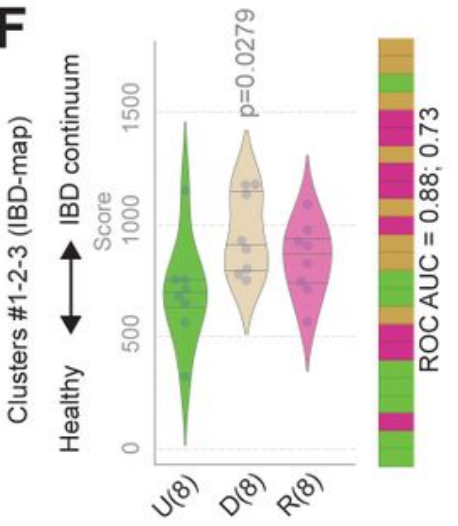

\section{G}

KEGGTIGHT-JUNCTION

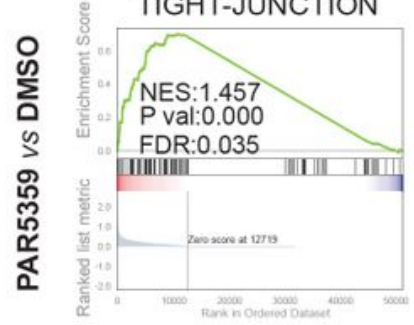

GO-ADHERENS-

COATES-MACROPHAGE-

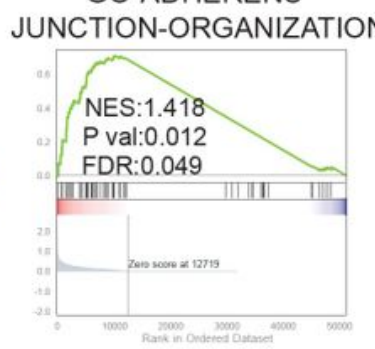
M1-VS-M2-UP

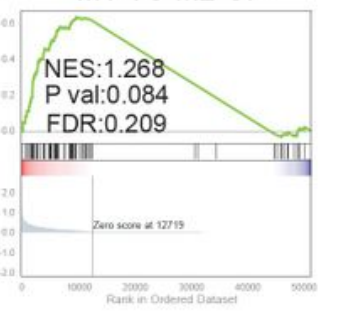




\section{Figure 3}

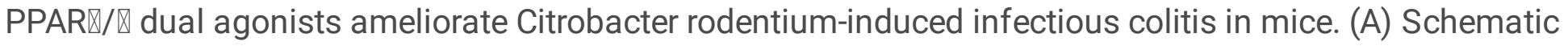
summarizing the workflow for testing PPAR-targeted therapeutics in C. rodentium-induced colitis. Mice were gavaged with $\mathrm{C}$. rodentium on day 0 and subsequently treated daily with PPAR agonists. Fecal pellets were collected to test viable bacterial burden, as determined by dilution plating and colony counting. Colons were excised on day 7 and 18 and analyzed using the indicated readouts. (B-D) Line graphs in B display time series of the burden of viable bacteria in feces. Scatter plots with bar graphs in C compare the peak burden of viable bacteria in feces on day 7. Scatter plots with bar graphs in $D$ display the area under the curve (AUC) for the line graph in B. (E) Images display representative fields from H\&Estained colon tissues. Mag $=100 x$ (top) and 200x (bottom). White arrowheads point to immune cell infiltrates. Statistics: All results are displayed as mean \pm SEM. Significance was tested using twoway/one-way ANOVA followed by Tukey's test for multiple comparisons. Significance: *, $p<0.05 ; * \star, p<$ 0.01 , ${ }^{* \star}, p<0.001$. (F) Violin plots (left) display the deviation of expression of genes in Clusters \#1-2-3 in the IBD network, as determined by RNA Seq on murine colons. Bar plot (right) displays the rank ordering of the samples. (G) Pre-ranked GSEA based on pairwise differential expression analyses (DMSO vs PAR5359 groups) are displayed as enrichment plots for epithelial tight (left) and adherens (middle) junction signatures and balanced macrophage processes (right). See also Supplementary Fig. 7 for the Day \#7 results in the C. rodentium-induced colitis model, Supplementary Fig. 8 for extended GSEA analyses, and Supplementary Fig. 9 for the effect of PAR5359 on DSS-induced colitis in mice. 
A

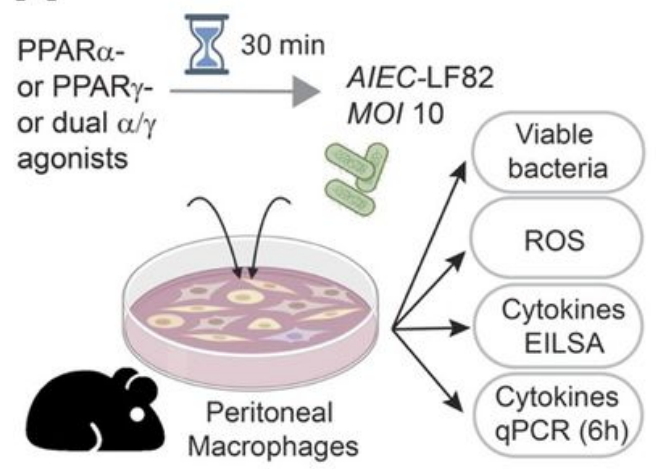

PPAR $\alpha$-agonist GW7647

PPAR $\gamma$-agonist Pioglitazone

PPAR $\alpha / \gamma$ - agonist PAR5359

B

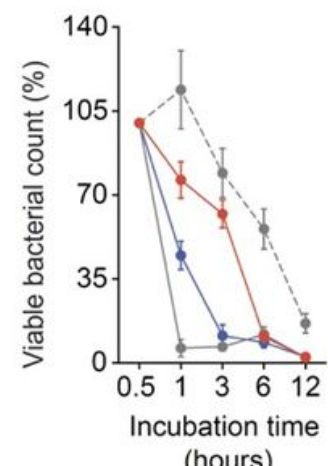

(hours)

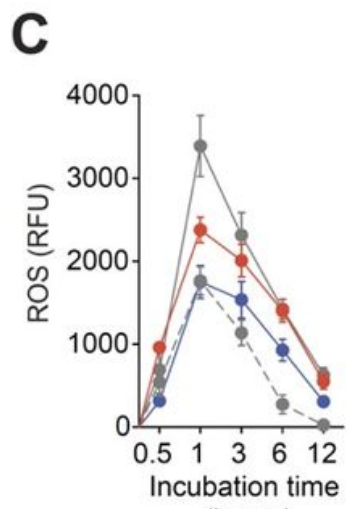

(hours)
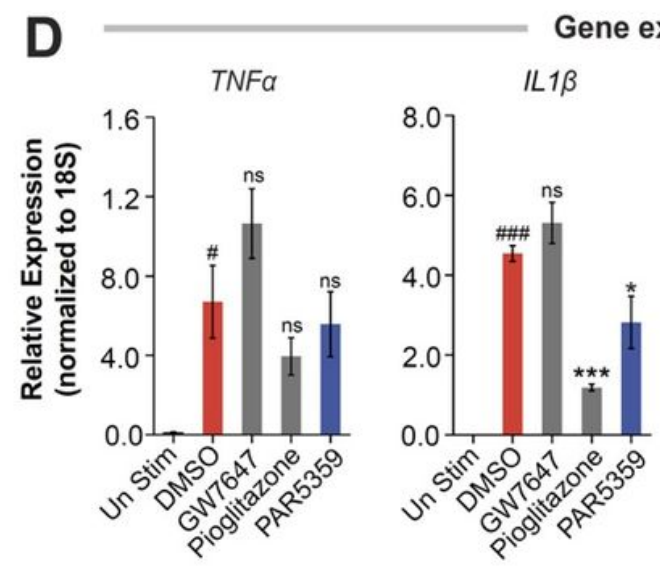

Gene expression
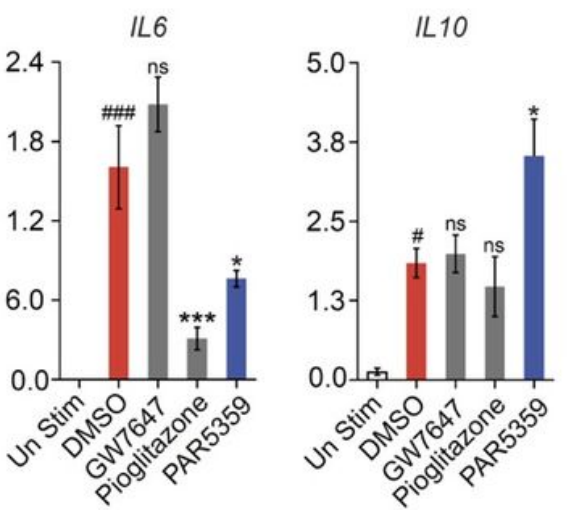

E

$T N F a$
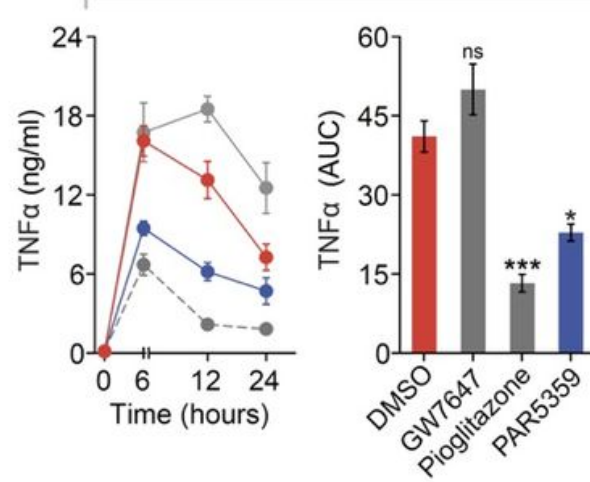

Protein expression

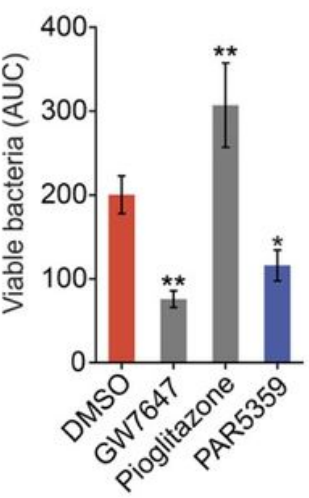

IL6
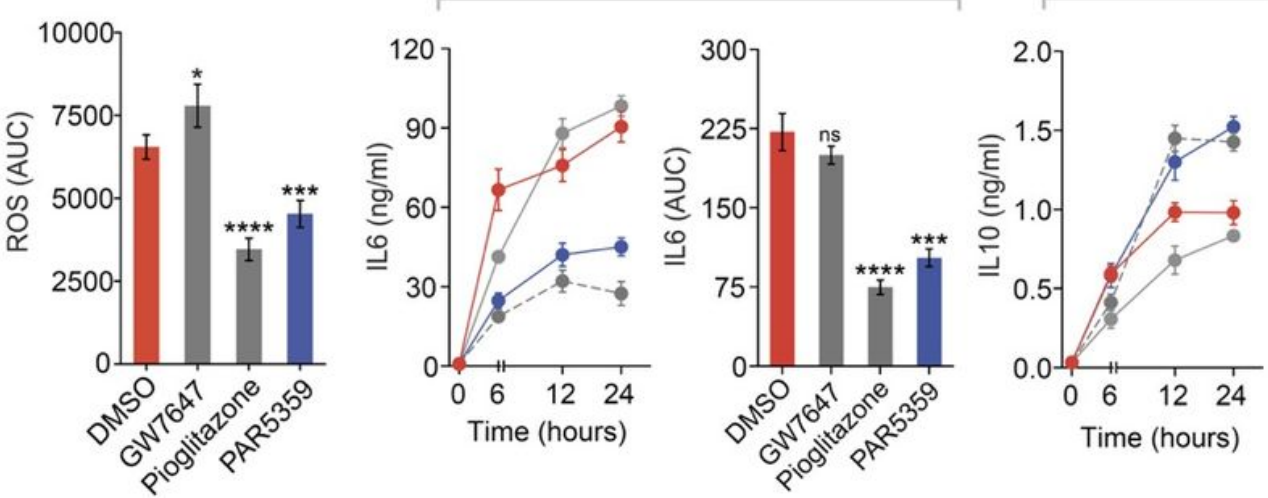

IL1 $\beta$
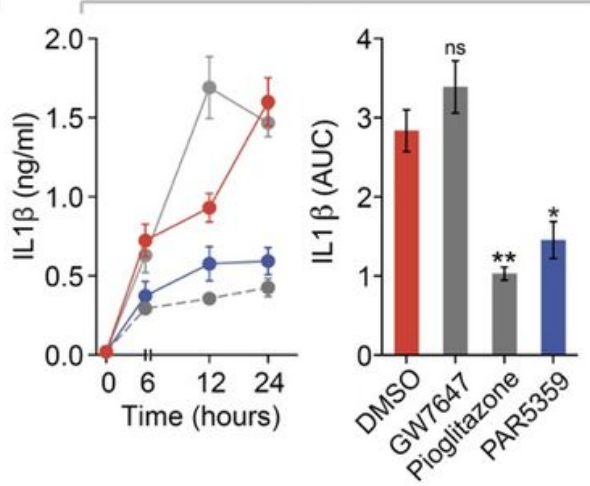

IL10
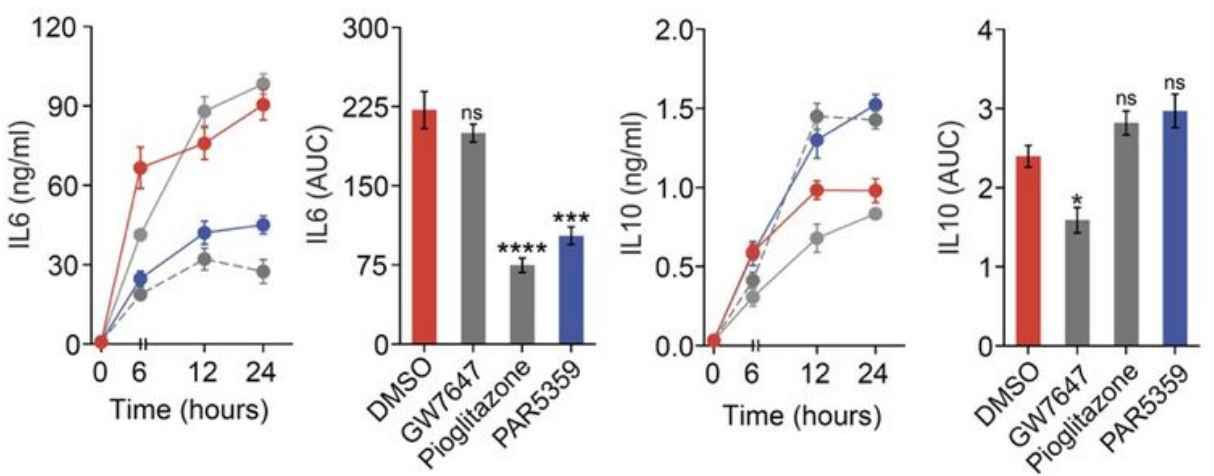

Figure 4

PPAR $\$ and PPAR $\ /$-dual agonists enhance, whereas PPAR $\$ agonist delay bacterial (AIEC LF82) clearance. (A) Schematic displays the experimental design and workflow. Thioglycolate-induced murine peritoneal macrophages (TG-PM) pretreated with PPAR agonists (see box, below; 20 nM GW7647, $10 \mu \mathrm{M}$ Pioglitazone and $1 \mu \mathrm{M}$ PAR5359) were infected with AIEC-LF82 (MOI 10) and subsequently analyzed for the bacterial count (Gentamycin protection assay), generation of cellular ROS, secretion of inflammatory cytokines (in supernatant media by ELISA) and the induction of cytokines (gene transcript analysis by qPCR). (B) Line graphs (left) display percent viable bacterial counts at indicated times after infection. Bar graphs (right) display the AUC. (C) Line graphs (left) and bar graphs (right) display the 
extent of ROS generation over time. (D) Bar graphs display the relative expression of transcripts of multiple cytokines (IL1 $\beta$, IL6, TNF囚 and IL10). (E) Line graphs (left) and bar graphs (right) showing the levels of secreted cytokines in the media. Statistics: All results are from at least three independent experiments and results displayed as means \pm SEM. Significance was tested using two-way/one-way ANOVA followed by Tukey's test for multiple comparisons. Significance: '\#' significance over uninfected TG-PMs and ' $*$ ' shows significance over AIEC-LF82 infected cells. ns, nonsignificant, *, $p<0.05 ; * *, p<$ $0.01, * \star \star, p<0.001, * \star \star \star, p<0.0001$. See Supplementary Fig. 10 for similar bacterial clearance assays performed using Salmonella enteritica.
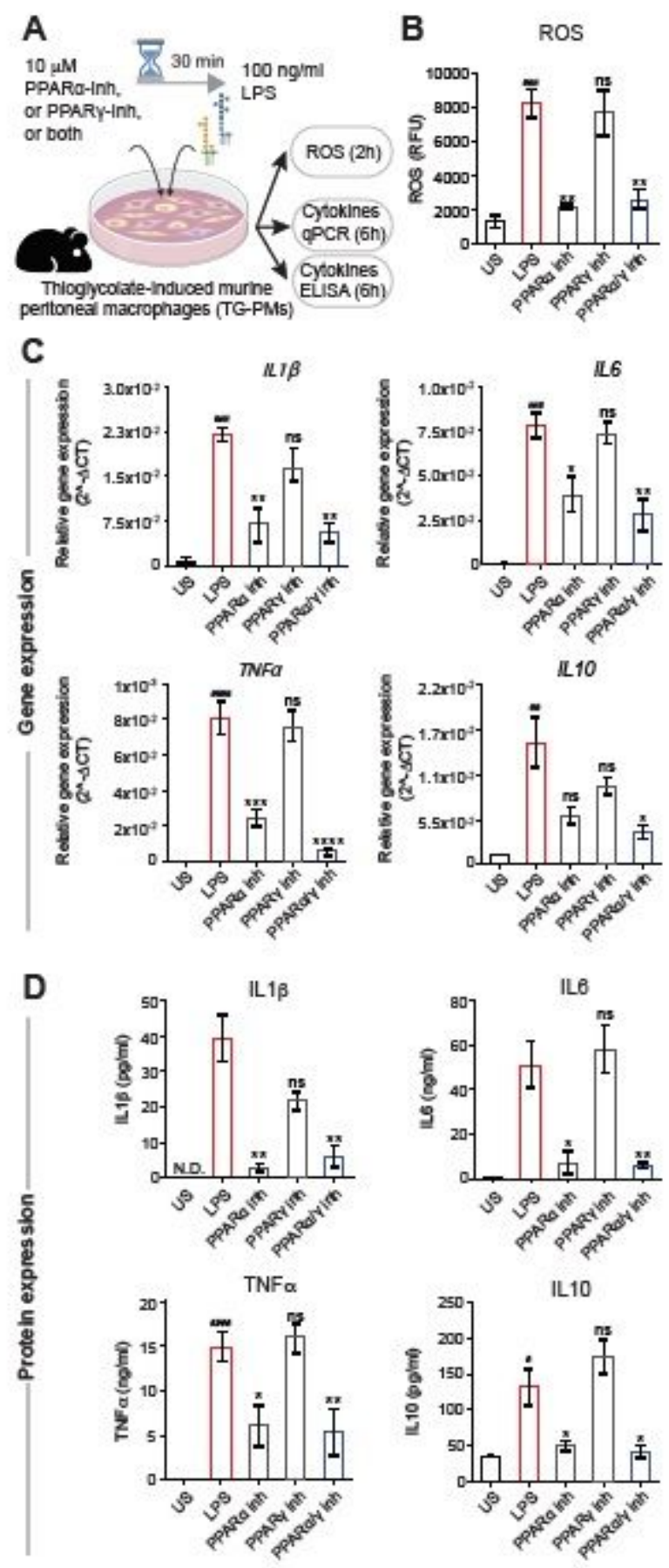

Figure 5 
PPARa but not PPARg is required for induction of cellular ROS and proinflammatory cytokines. (A) Schematic of experimental design. TG-PMs were pre-incubated with $10 \mu \mathrm{M}$ PPARa or PPARg inhibitors, either alone or in combination for $30 \mathrm{~min}$ prior to stimulation with $100 \mathrm{ng} / \mathrm{ml}$ LPS. Cells were analyzed at 2 and $6 \mathrm{~h}$ to estimate cellular ROS and cytokine induction, respectively. (B-D) Bar graphs display the levels of cellular ROS (B), relative levels of mRNA (C) and protein (D) expression of cytokines (IL1 $\beta$, IL6, TNFV and IL10). Statistics: Results are from three independent experiments and displayed as mean \pm SEM. One-way ANOVA followed by Tukey's test for multiple comparisons was performed to test significance. Significance: ns: nonsignificant, $*, p<0.05 ; * \star, p<0.01, * \star \star, p<0.001$ and $* \star \star \star, p<$ 0.0001.PPARa but not PPARg is required for induction of cellular ROS and proinflammatory cytokines. (A) Schematic of experimental design. TG-PMs were pre-incubated with $10 \mu \mathrm{M}$ PPARa or PPARg inhibitors, either alone or in combination for $30 \mathrm{~min}$ prior to stimulation with $100 \mathrm{ng} / \mathrm{ml}$ LPS. Cells were analyzed at 2 and $6 \mathrm{~h}$ to estimate cellular ROS and cytokine induction, respectively. (B-D) Bar graphs display the levels of cellular ROS (B), relative levels of mRNA (C) and protein (D) expression of cytokines (IL1 $\beta$, IL6, TNFV and IL10). Statistics: Results are from three independent experiments and displayed as mean \pm SEM. One-way ANOVA followed by Tukey's test for multiple comparisons was performed to test significance. Significance: ns: nonsignificant, $*, p<0.05 ; * \star, p<0.01, * \star *, p<0.001$ and ${ }^{* \star \star *}, p<0.0001$. 
A
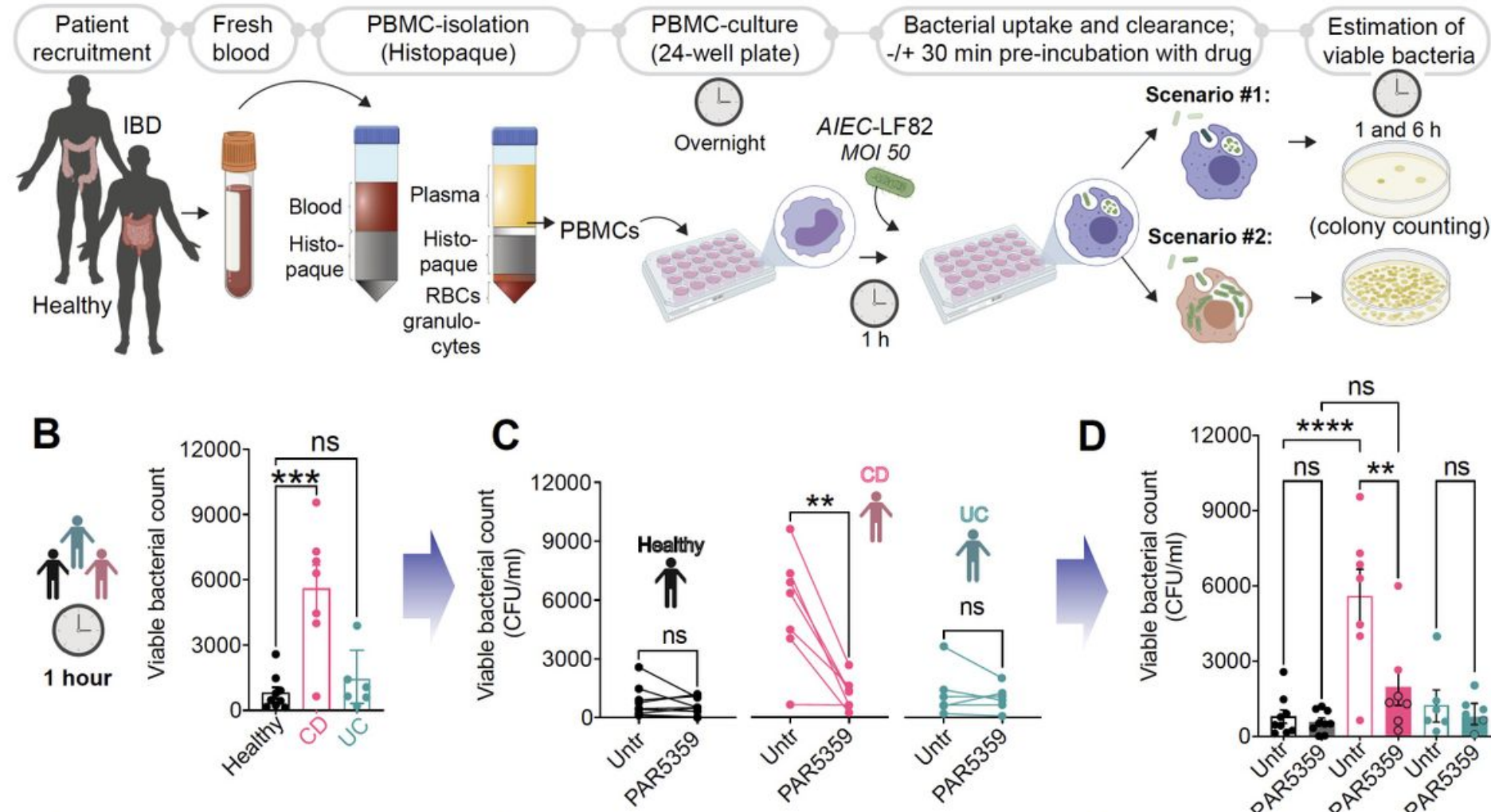

E

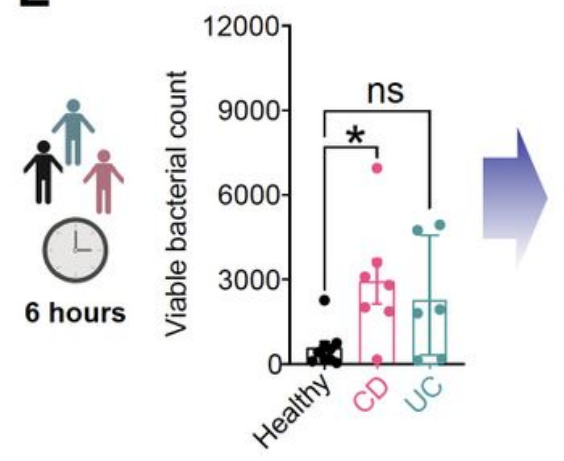

\section{$\mathbf{F}$}

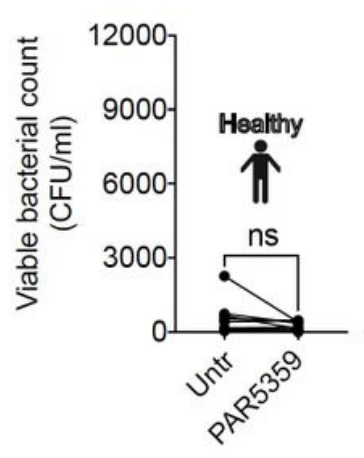

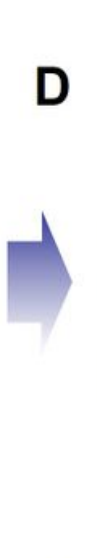

G
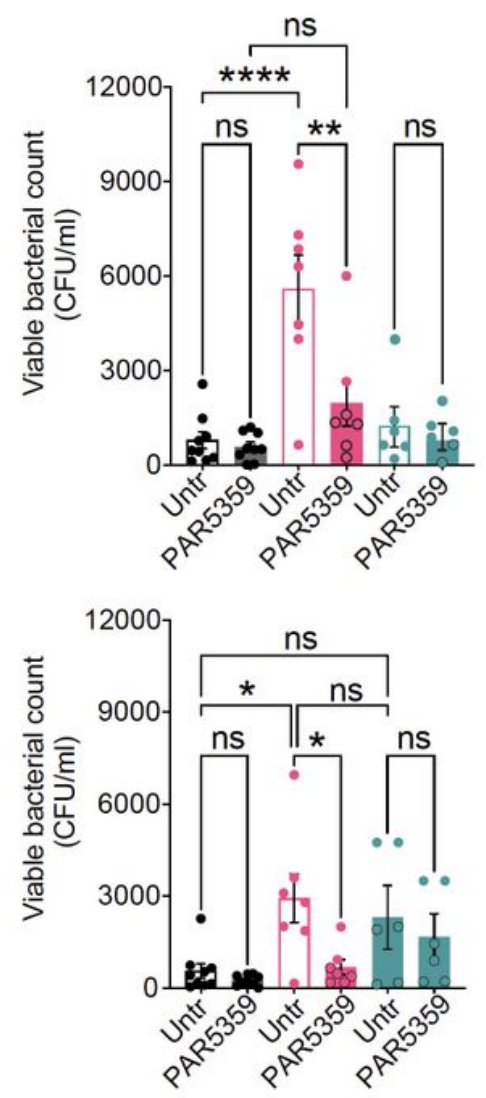

Figure 6

PPARa/g dual agonist, PAR5359, promotes the clearance of AIEC-LF82 from CD patient-derived PBMCs. (A) Schematic displays the overall experimental design using human subjects (see Table 5 for patient demographics). Peripheral blood collected from healthy, CD and UC patients was used as a source of PBMCs. PBMCs were pre-treated for 30 min with $1 \mu \mathrm{M}$ PPARa/g agonists prior to infection with AIEC-LF82 (MOI 50) for 1h. PBMCs were subsequently treated with gentamicin to kill extracellular microbes for $60 \mathrm{~min}$ ( $\sim \mathrm{t0} \mathrm{h}$ ) prior to lysis and plating to determine the intracellular abundance of viable bacteria at $\mathrm{t} 1 \mathrm{~h}$ and $\mathrm{t} 6 \mathrm{~h}$, as determined by dilution plating and colony counts (see Methods for details). Bar graphs with scatter plot display the abundance of viable intracellular bacteria at $1 \mathrm{~h}(\mathrm{~B})$ and $6 \mathrm{~h}(\mathrm{E})$ after infection. Paired line plots display the rate of clearance of bacteria in individual subjects at $1 \mathrm{~h}(\mathrm{C})$ and $6 \mathrm{~h}(\mathrm{~F})$ after infection. Data in B-C of $1 \mathrm{~h}$ infection is combined in (D) and data from $E$ and $F$ 6h infection is combined in $(G)$ with statistics: Results are displayed as mean \pm SEM (CD patient $n=7$, UC 
patients $=6$ and healthy $n=9$ ). Paired t-test or One-way ANOVA followed by Tukey's test for multiple comparisons was performed to test significance. Significance: ns: non-significant, $*, p<0.05 ; * \star, p<0.01$, $* * *, p<0.001$ and $* * * *, p<0.0001$.

\section{Network-rationalized target identification}

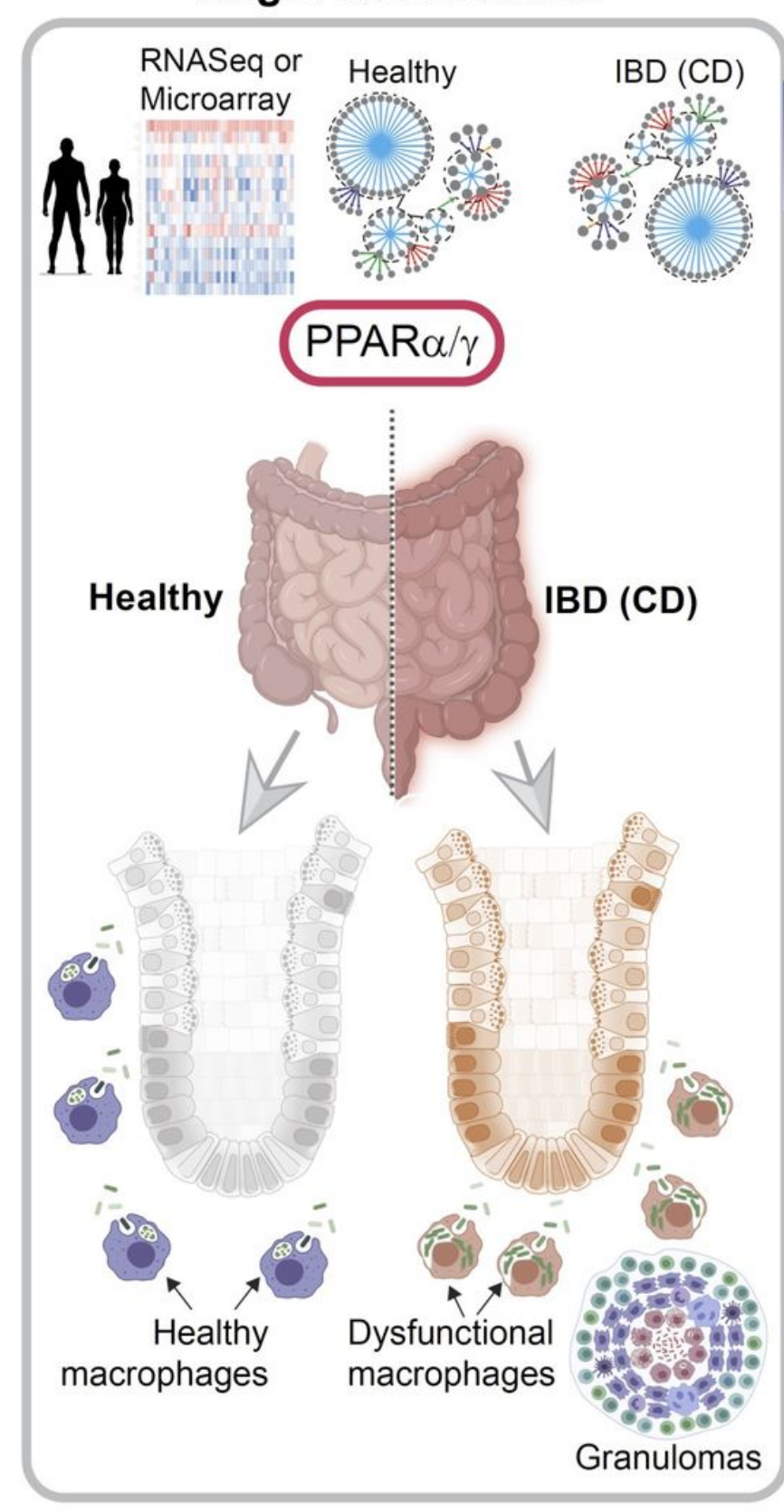

\section{Experimental determination of mechanism of action}

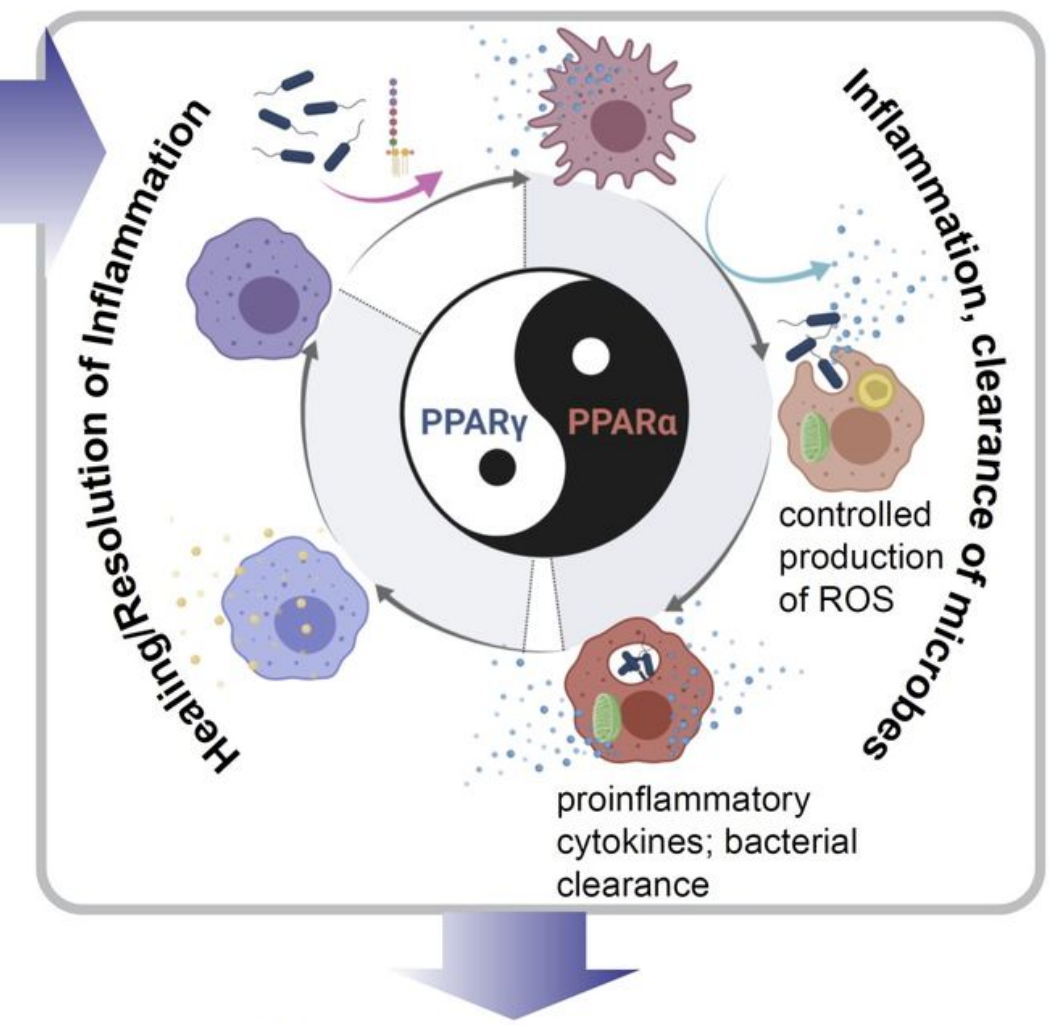

\section{Validation in pre-clinical models}

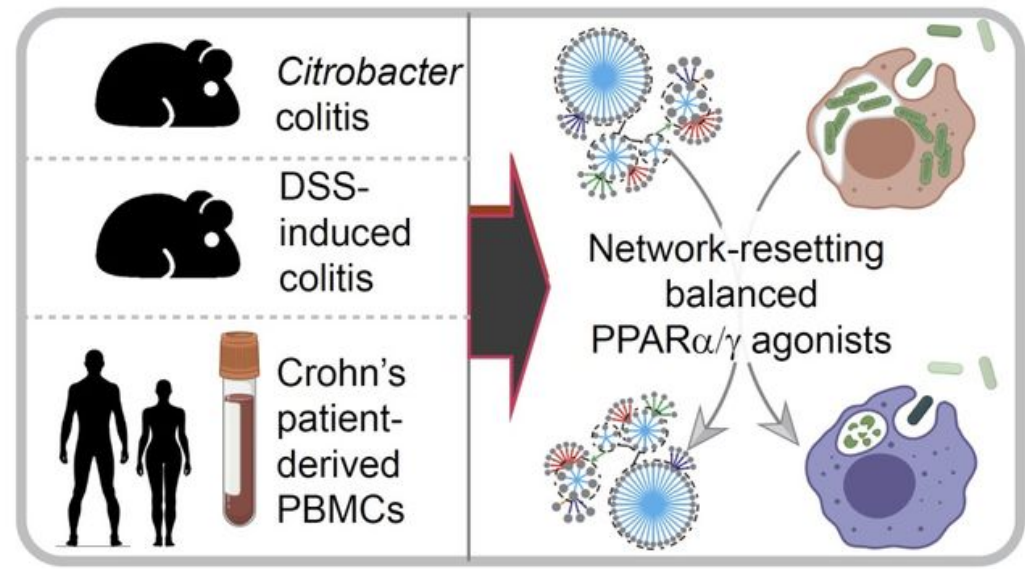

Figure 7

Summary of findings and working model Schematic summarizes key approaches and findings of this study. First, network-rationalized target identification (Left) was performed using web-based platform that queries > 1000 IBD datasets [9; see Methods] that served as 'input' to create a map of gene clusters that are progressively altered in the gut in the setting of IBD. Predictions are used to guide the choice of therapeutics (dual agonists of PPARa and PPARg that have a balanced agonistic potential for both 
PPARs), the choice of animal models of IBD, predict cell types of action (macrophage processes), and finally, the subtype of IBD that could benefit most based on the cell type of action (i.e., CD). Second, experimentally determined mechanism of action studies (right, top) showed that balanced actions of both PPARa and PPPARg enable the induction of bacterial clearance, resolution of inflammation and healing; PPARa is responsible for ROS and cytokine induction, whereas PPPARg is responsible for antiinflammatory response and healing. The dual agonistic action was superior to each agonist used alone. Third, targets validation studies (right, bottom) in murine and human models confirm the use of PPARa/g dual agonists for enhancing bacterial clearance and protection against colitis. When tested side-by-side in the infectious colitis model, the dual agonistic action was superior to each agonist used alone.

\section{Supplementary Files}

This is a list of supplementary files associated with this preprint. Click to download.

- nrreportingsummaryFILLED.pdf

- 2.Supplementaryonlinematerials.pdf 